\title{
Beam commissioning of the 3-GeV rapid cycling synchrotron of the Japan Proton Accelerator Research Complex
}

\author{
H. Hotchi, ${ }^{*}$ M. Kinsho, K. Hasegawa, N. Hayashi, Y. Hikichi, S. Hiroki, J. Kamiya, K. Kanazawa, M. Kawase, F. Noda, ${ }^{\dagger}$ \\ M. Nomura, N. Ogiwara, R. Saeki, P. K. Saha, A. Schnase, Y. Shobuda, T. Shimada, K. Suganuma, H. Suzuki, \\ H. Takahashi, T. Takayanagi, O. Takeda, F. Tamura, N. Tani, T. Togashi, T. Ueno, M. Watanabe, Y. Watanabe, \\ K. Yamamoto, M. Yamamoto, Y. Yamazaki, H. Yoshikawa, and M. Yoshimoto \\ Japan Atomic Energy Agency (JAEA), Tokai, Naka, Ibaraki, 319-1195, Japan
}

\author{
A. Ando, H. Harada ${ }^{\ddagger}$ Y. Irie, C. Ohmori, K. Satou, Y. Yamazaki, and M. Yoshii \\ High Energy Accelerator Research Organization (KEK), Tsukuba, Ibaraki, 305-0801, Japan
}

(Received 2 December 2008; published 30 April 2009)

\begin{abstract}
The 3-GeV rapid cycling synchrotron (RCS) of the Japan Proton Accelerator Research Complex (J-PARC) was commissioned in October 2007, and successfully accomplished $3 \mathrm{GeV}$ acceleration on October 31. Six run cycles through February 2008 were dedicated to commissioning the RCS, for which the initial machine parameter tuning and various underlying beam studies were completed. Then since May 2008 the RCS beam has been delivered to the downstream facilities for their beam commissioning. In this paper we describe beam tuning and study results following our beam commissioning scenario and a beam performance and operational experience obtained in the first commissioning phase through June 2008.
\end{abstract}

DOI: 10.1103/PhysRevSTAB.12.040402

PACS numbers: 29.20.dk, 29.27.Ac, 29.27.Fh, 29.27.Bd

\section{INTRODUCTION}

The Japan Proton Accelerator Research Complex (JPARC) located on the campus of Japan Atomic Energy Agency (JAEA) in Tokai, Naka, Japan, which was formed by combining the neutron science project of JAEA and the hadron facility project of High Energy Accelerator Research Organization (KEK), is a multipurpose proton accelerator facility aiming at MW-class output beam power. The J-PARC accelerator complex [1] comprises a 400-MeV linac, a 3-GeV rapid cycling synchrotron (RCS), a 50-GeV main ring synchrotron (MR), and several experimental facilities (a materials and life science experimental facility; MLF, a hadron experimental hall, and a neutrino beam line to Kamioka).

The J-PARC beam commissioning started in November 2006 and it has well proceeded as planned from the linac toward the downstream facilities, the RCS, and then the MR and MLF so far. We had 18 running periods in total through June 2008. A typical run cycle consists of two- or three-week beam operation with oneweek maintenance and service break. The first eight run cycles through June 2007 were dedicated to commissioning the linac. The initial linac beam tuning to secure the beam quality required for the coming RCS commissioning was completed in these periods [2,3]. After the scheduled

\footnotetext{
*hotchi.hideaki@jaea.go.jp

†resent address: Energy and Environmental Systems Laboratory, Hitachi, Ltd., Hitachi, Ibaraki, 319-1221, Japan.

${ }^{\ddagger}$ Also at Graduate School of Science, Hiroshima University, Higashihiroshima, Hiroshima, 739-8526, Japan.
}

summer shutdown (July-August), the RCS was commissioned in October 2007 and successfully accomplished $3 \mathrm{GeV}$ acceleration on October 31 . Six run cycles through February 2008 were dedicated to RCS commissioning, for which we completed the initial beam tuning and lots of data taking essential for estimating a basic performance of the RCS. Then since May 2008 the extracted RCS beam has been delivered to the MR and MLF for their beam commissioning [4]. Since then, while the priority has been given to MR and MLF beam tuning, the RCS also continues further beam tuning and studies toward higher beam intensity. Now the RCS is in transition from the first commissioning phase to the next challenging stage, and our efforts hereafter will be focused on higher power operations. In this paper, we describe beam tuning and study results following our actual beam tuning procedure and a beam performance and operational experience obtained in the first stage of the RCS beam commissioning (eight run cycles from October 2007 through June 2008).

\section{DESCRIPTION OF THE RCS}

Figure 1 shows a schematic view of the RCS. The RCS has two functions as a proton driver for the MLF and as an injector to the MR. A negative hydrogen ion $\left(\mathrm{H}^{-}\right)$beam from the linac is delivered through the linac-to-3-GeV beam transport line (L-3BT) to the RCS injection point, where it is multiturn charge-exchange injected with a carbon stripper foil. The RCS accelerates the injected beam up to $3 \mathrm{GeV}$ at $25 \mathrm{~Hz}$ repetition. With the current injection energy of $181 \mathrm{MeV}$, the RCS aims at providing 


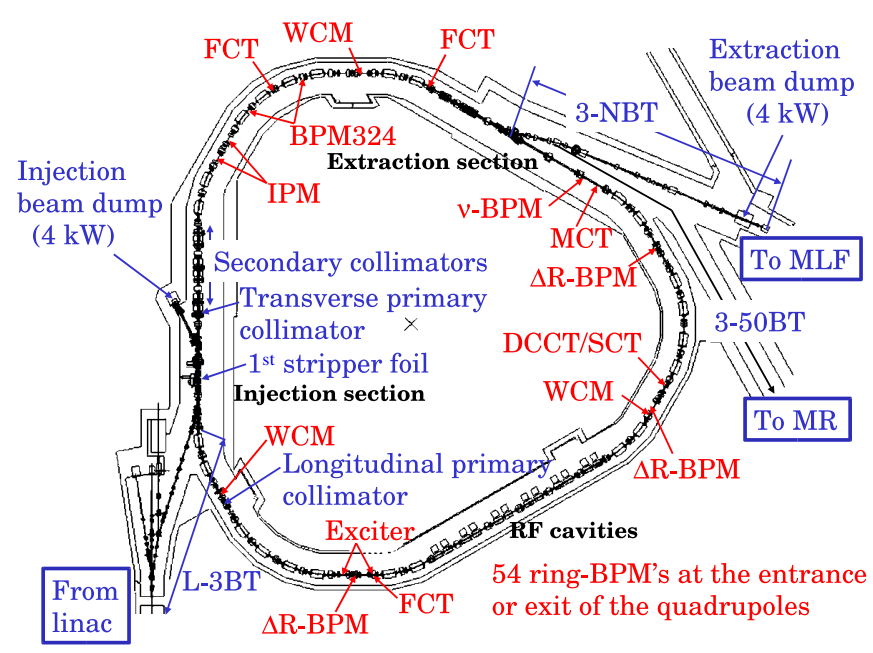

FIG. 1. (Color) Schematic view of the RCS, where the red characters correspond to the beam diagnostics devices which are defined in Table II.

0.3-0.6 MW output beam power. After upgrading the linac energy to $400 \mathrm{MeV}$ by adding an annular coupled structure (ACS) linac [5] in the future, the RCS will aim at $1 \mathrm{MW}$ output. Most of the time, the $3 \mathrm{GeV}$ beam from the RCS is transported via the 3-GeV-to-neutron-target beam transport line (3-NBT) to the MLF to produce pulsed spallation neutrons and muons. A part of the RCS beam (typically four pulses every $3.64 \mathrm{~s}$ ) is transported via the $3-\mathrm{GeV}$-to$50-\mathrm{GeV}$ beam transport line (3-50BT) to the MR.

The RCS has a threefold symmetric lattice over its circumference of $348.333 \mathrm{~m}$. The RCS focusing structure consists of 24 dipole magnets (one family called BM) and 60 quadrupole magnets (seven families called QFL, QDL, QFM, QDX, QFN, QDN and QFX, where QF and QD mean horizontally and vertically focusing quadrupoles), which are excited with a $25 \mathrm{~Hz}$ DC-biased sinusoidal current pattern in eight independent resonant networks [6,7]. In addition, 26 sets of horizontal (26 sets of vertical) steering magnets are installed close to the QF (QD) quadrupole magnets for global and local corrections of the closed orbit. Each superperiod consists of two 3-DOFO arc modules and a 3-DOFO long straight insertion, where DOFO means a defocusing-focusing periodic cell. Each arc module has a missing-bend cell, where the horizontal dispersion has a maximum (around $5 \mathrm{~m}$ ). Such a lattice structure was adopted to separate the transition energy (higher than $9 \mathrm{GeV}$ ) far enough from the extraction energy. Three families of sextupole magnets utilized for the chromatic correction and a longitudinal primary collimator (scatterer) are installed there. The straight insertions have no dispersion. Injection [8] and collimation [9] systems are installed in the first straight section; the injection system uses the first one and a half cells, and the transverse primary collimator (scatterer) and the secondary collimators (absorber) take the rest. Extraction [8] and rf [10]
TABLE I. RCS design parameters.
Circumference

Superperiodicity

Injection energy

Extraction energy

Repetition rate

Ramping pattern

Injection period

Harmonic number

Number of bunches

Output beam power

Number of particles per pulse

Design betatron tune $\left(\nu_{x} / \nu_{y}\right)^{\mathrm{e}}$

Natural chromaticity $\left(\xi_{x} / \xi_{y}\right)^{\mathrm{f}}$

Transition energy

Momentum acceptance

Ring acceptance

Collimator acceptance

Transverse painting emittance

Longitudinal beam emittance

Bunching factor at injection

Space-charge tune shift

at injection for $0.3 \mathrm{MW}$ output

Number of ring magnets

- dipole magnets

- quadrupole magnets

- sextupole magnets

- steering magnets

Number of rf cavities

Number of pulsed elements

- horizontal pulse dipole magnets

for the injection-orbit bump

- horizontal/vertical

pulse dipole magnets for

the transverse painting injection

- extraction pulse kickers
$348.333 \mathrm{~m}$

3

$181 \mathrm{MeV}^{\mathrm{a}}$

$3 \mathrm{GeV}$

$25 \mathrm{~Hz}$

Sinusoidal

2

2

$0.3-0.6 \mathrm{MW}^{\mathrm{c}}$

$2.5-5.0 \times 10^{13 \mathrm{~d}}$

$6.68 / 6.27$

$-10 /-7$

$9.21 \mathrm{GeV}$

$\pm 1 \%$

$486 \pi \mathrm{mm} \operatorname{mrad}$ $324 \pi \mathrm{mm}$ mrad $216 \pi \mathrm{mm}$ mrad $2.5 \mathrm{eV} \mathrm{s}$

0.4

$-0.14^{\mathrm{g}}$

24

60 with 7 families

18 with 3 families

52

$12^{\mathrm{h}}$ (3 gaps per cavity)

4 (connected in series)

$4 / 2$

8 $0.5 \mathrm{~ms}(235 \text { turns })^{\mathrm{b}}$

${ }^{\mathrm{a}}$ It will be upgraded to $400 \mathrm{MeV}$ by adding an ACS linac section.

${ }^{\mathrm{b}}$ It is $0.5 \mathrm{~ms}$ (307 turns) for $400 \mathrm{MeV}$ injection energy.

${ }^{\mathrm{c}}$ The design output beam power for $400 \mathrm{MeV}$ injection energy is $1 \mathrm{MW}$.

${ }^{\mathrm{d}}$ It is $8.3 \times 10^{13}$ for $1 \mathrm{MW}$ output.

${ }^{\mathrm{e}}$ It means horizontal/vertical tunes.

${ }^{\mathrm{f}}$ It means horizontal/vertical chromaticities.

${ }^{\mathrm{g}}$ It is comparable at $400 \mathrm{MeV}$ injection energy for $1 \mathrm{MW}$ output.

${ }^{\mathrm{h}} \mathrm{The}$ current number is 10 . Another two rf cavities will be installed in the future.

systems are located in the second and third straight section, respectively.

The RCS design parameters are listed in Table I. One of the key issues to achieve such a high-intensity operation is to control and localize beam losses and decrease uncontrolled beam losses. In high-intensity proton machines like the RCS, the space-charge effect would pose a severe limitation on the achievable beam intensity. Namely, various resonances driven by the space-charge force of the beam and in combination with cyclic external fields can 
be one of the main sources of beam emittance growths and particle losses. In order to mitigate the space-charge effect, the RCS adopts a multiturn painting injection scheme in both the transverse and longitudinal phase spaces. An adequate ratio between physical and collimator apertures is another important factor to localize beam losses. In the RCS, the transverse painting emittance and the transverse collimator acceptance are each set at $216 \pi$ and $324 \pi \mathrm{mm}$ mrad in the design, for which the ring acceptance of $486 \pi \mathrm{mm}$ mrad is secured for a possible momentum spread of $\pm 1 \%$ to keep a sufficient collimation efficiency of better than $98 \%$ [11]. While such a large acceptance is essential for high-intensity machines, its resultant large-gap magnets may carry some disadvantages. Especially in compact machines like the RCS, its aspect ratio (inner diameter over magnet length) is obviously large, consequently causing a large nonlinear fringe field. Such nonlinear magnetic fields excite different highorder transverse resonances, causing a shrinkage of the dynamic aperture $[12,13]$. Such a situation will be more critical for a higher-intensity beam. The nonlinearity of the space-charge force, especially the octupole component, is another strong driving force for nonlinear resonances. In addition, the defocusing property of the space-charge force causes a large incoherent tune shift. These would further complicate the stability and flexibility of the betatron tune space. These circumstances also will force a complex optimization procedure for the beam painting scheme, because nonlinear effects are enhanced by larger amplitudes of the transverse and longitudinal oscillations. For this kind of machine where various beam-intensity dependent nonlinear effects are complicatedly intertwined, clearly understanding the basic accelerator performance by looking at fundamental single-particle behavior with a low-intensity and narrow beam is a key issue at the first commissioning phase. Of course, such a low current beam is also required to avoid unnecessary beam losses at the initial stage of the beam tuning. The beam intensity thereafter will be increased gradually through a step-by-step parameter optimization with carefully monitoring a trend of the residual radiation levels of the accelerator components.

\section{BEAM DIAGNOSTICS SYSTEM}

The beam diagnostics system is a key apparatus to understand a behavior of the beam and to perform the beam tuning procedure efficiently. As listed in Table II, various beam diagnostics devices have been prepared for many uses [14].

Seven sets of scan-type multiwire profile monitors (MWPM1-7 [15,16]), which are installed in the injection section, are utilized to estimate the Twiss parameters and transverse emittances of the injection beam as well as to establish the injection beam orbit. The MWPM4 has two wire sensor frames, where the wires are put with 3 and $10 \mathrm{~mm}$ pitches parallel to the $\mathrm{U}$ and $\mathrm{V}$ axes 17.7 degrees rotated from the horizontal and vertical axes on the transverse two-dimensional plane. In order to obtain a better

TABLE II. Beam diagnostics system employed in the RCS beam commissioning.

\begin{tabular}{|c|c|c|c|}
\hline & Name & Number & Main use \\
\hline Scan type of multiwire profile monitor & MWPM & 7 & Transverse profile of the injection beam \\
\hline \multicolumn{4}{|l|}{ Beam position monitor (BPM) } \\
\hline \multirow[t]{3}{*}{ - Electrostatic type of BPM } & Ring-BPM & 54 & Closed orbit and turn-by-turn beam position \\
\hline & $\Delta \mathrm{R}-\mathrm{BPM}$ & 3 & Rf radial feedback \\
\hline & $\nu$-BPM & 1 & Betatron tune \\
\hline - Strip-line type of BPM & $\begin{array}{c}\text { K-BPM, I-BPM, } \\
\text { and BPM324 }\end{array}$ & 4 & Single-pass orbit of the injection beam \\
\hline Residual gas ionization profile monitor & IPM & 2 & Turn-by-turn transverse beam profile \\
\hline \multicolumn{4}{|l|}{ Current transformer (CT) and bunch monitor } \\
\hline - Direct current CT/slow CT & $\mathrm{DCCT} / \mathrm{SCT}$ & $1 / 1$ & Average current of the circulating beam \\
\hline - Medium CT & MCT & 1 & Charge accumulation process during injection \\
\hline - Fast CT/wall current monitor & FCT/WCM & $3 / 3$ & $\begin{array}{c}\text { Longitudinal bunch shape, } \\
\text { rf phase feedback, and } \\
\text { rf voltage feedforward for } \\
\text { beam loading compensation }\end{array}$ \\
\hline \multicolumn{4}{|l|}{ Beam loss monitor } \\
\hline - Scintillator with photomultiplier & S-BLM & 24 & Beam loss detection \\
\hline - Coaxial type of air ionization chamber & AIC-BLM & 20 & Same \\
\hline - Proportional chamber with $\mathrm{Ar}-\mathrm{CO}_{2}$ & P-BLM & 90 & Same (connected to MPS) \\
\hline
\end{tabular}


resolution, the wire frames are horizontally scanned for $10 \mathrm{~mm}$ typically with $0.1 \mathrm{~mm}$ step. The other MWPM's also have similar wire configurations.

Three kinds of electrostatic-type beam position monitors (BPM) are prepared for various uses [14]. The first one is for both closed orbit and turn-by-turn beam position measurements (ring-BPM $[17,18]$ ). For this purpose, 54 sets of ring-BPM's are distributed at the entrance or exit of the ring quadrupole magnets. The second one is for the rf radial feedback $(\triangle \mathrm{R}-\mathrm{BPM})$. In order to get a better sensitivity for the orbit shift coming from a momentum mismatch, three sets of $\triangle$ R-BPM's with only two electrodes, which are sensitive for the horizontal direction, are installed in the missing-bend cells with dispersion maximum. The last one is for the betatron tune measurement $(\nu$-BPM). In this measurement, two sets of exciters, which give dipole kicks for the horizontal and vertical planes, are utilized to excite coherent betatron oscillations. The signal detected at $\nu$-BPM is processed by a real-time spectrum analyzer. In addition, two sets of strip-line type BPM's are each installed most downstream of L-3BT (K and I-BPM) and in the first arc section of the ring (BPM324) [14]. They are for observing the single-pass orbit of the injection beam and tuned to detect $324 \mathrm{MHz}$ linac rf frequency.

In order to obtain the turn-by-turn transverse beam profile, two sets of residual gas ionization profile monitors (IPM [19]) for the horizontal and vertical planes are installed in the first arc section of the ring. Each IPM consists of three microchannel plates (MCP) for charge signal multiplication and signal readout, electron sources to check gain balance of MCP's, electrodes for high voltage feeder, and a 3-pole magnet to generate guiding field. While IPM's are equipped with both functions of ion and electron corrections, we have mainly used them with the ion correction mode with no guiding field so far.

Several kinds of current transformers (CT) and bunch monitors are installed [14]; a direct current CT (DCCT) and a slow CT (SCT) for measuring the average current of the circulating beam, a medium CT (MCT) for observing the charge accumulation process during the multiturn injection, and three fast CT's (FCT) and three wall current monitors (WCM) for observing the longitudinal bunch shape, in which two of FCT's and one of WCM's are reserved for the rf phase feedback and the rf voltage feedforward for beam loading compensation.

In order to observe the beam loss distribution during operation, three kinds of beam loss monitors (BLM [20]) are distributed in the entire RCS; 24 sets of scintillators, each of which is equipped with a photomultiplier (SBLM), 20 sets of coaxial type of air ionization chambers (AIC-BLM), and 90 sets of proportional chambers with Ar- $\mathrm{CO}_{2}$ mixture gas (P-BLM). In these, the output signals of P-BLM's are connected to the machine protection system (MPS [21]) which promptly turns off the beam operation when the beam loss monitor signals surpass a threshold which we set.
On the basis of the above beam diagnostics system available at the initial commissioning phase, we carefully made the best possible beam commissioning scenario including several alternative ways.

\section{BEAM CONDITION AT THE INITIAL BEAM TUNING PHASE}

The linac beam comprises chopped pulses synchronized to the ring $\mathrm{rf}$ frequency (around $938 \mathrm{kHz}$ ) at the injection time. At full capability in the current configuration, the linac will produce $36 \mathrm{~kW}$ output at $181 \mathrm{MeV}$ with $30 \mathrm{~mA}$ peak, $0.5 \mathrm{~ms}$ long, and $53 \%$ chopper beam-on duty factor at $25 \mathrm{~Hz}$ repetition, which corresponds to $0.6 \mathrm{MW}$ output at the RCS. The residual beam current in gaps between the chopped pulses with $25 \mathrm{~mA}$ peak current was experimentally estimated to be much less than $10^{-3}$ of the peak by kicking out the entire linac beam pulse with the chopper system.

The number of particles per bunch of the RCS is basically adjusted by combination of the peak current and pulse length. The momentum spread of the RCS beam is also adjustable by controlling the chopped-pulse width. Most of the initial beam tuning phase of the RCS was performed with low-power and low-duty operations so that the beam loss and also the space-charge induced effect did not cause concern; around $4.2 \times 10^{11}$ particles per bunch in the single-bunch operation ( $\mathrm{rf}$ harmonic number $h=2$ ) typically using a linac beam with $5 \mathrm{~mA} / 0.05 \mathrm{~ms} / 26 \%$ or $25 \mathrm{~mA} / 0.05 \mathrm{~ms} / 5 \%$ (peak current/pulse length/chopper beam-on duty factor). As to the beam repetition, beamon-demand operation (with no beam repetition) or $1 \mathrm{~Hz}$ operation if necessary were mainly employed in the initial beam tuning period.

The transverse emittance of the injection beam (normalized, in rms) was obtained in the transverse matching at L-3BT to be $0.17 / 0.17 \pi \mathrm{mm}$ mrad ( $5 \mathrm{~mA}$ peak current) and $0.25 / 0.25 \pi \mathrm{mm} \mathrm{mrad}$ ( $25 \mathrm{~mA}$ peak current) for the horizontal/vertical planes [22]. As shown in Fig. 3 later, the linac beam has a non-Gaussian tail component probably coming from residual transverse and longitudinal mismatches in the linac matching sections [3]. While it is not critical for the low-intensity beam employed in the initial tuning phase, a further fine-tuning to suppress such a halo formation will be essential to fulfill more flexible transverse painting injection in aiming at higher beam intensities. The momentum spread of the linac beam was evaluated to be $0.15 \%$ in full width from the debunching time measured with no ring of in the RCS beam storage mode.

The stability of the linac beam, namely, its energy and beam position jitters monitored over several hours were less than $20 \mathrm{keV}$ (rms) and $0.06 \mathrm{~mm}$ (rms) at the RCS injection including intrinsic jitters of the monitor system [2]. Thus, the linac beam was very reproducible every 
pulse. This enabled us to perform the initial beam tuning efficiently with a minimum number of beam pulses to minimize unnecessary machine activation at the first beam commissioning phase.

\section{RCS OPERATION MODES}

Our beam tuning scenario comprises the following four operation modes: (i) injection beam dump mode, (ii) single-pass extraction mode, (iii) beam storage mode, and (iv) acceleration mode.

In the injection beam dump mode (i), the linac beam is directly driven to the injection beam dump. In this mode the tuning of the injection and dump-line orbits and also the evaluation of Twiss parameters for the injection beam were performed.

In the single-pass extraction mode (ii), the injected beam is immediately extracted after passing through one-third of the RCS circumference and driven to the extraction beam dump. This mode was utilized for roughly checking polarities of the ring magnets and a matching between the ring dipole magnetic field strength and the injection beam momentum at the beginning of the beam commissioning, and also for the transverse matching and orbit tuning of the injection beam likewise as the injection beam dump mode.

In the beam storage mode (iii), the RCS is operated like a storage ring at the injection energy. Our beam tuning scenario is to tune up the basic ring parameters such as optical parameters in the beam storage mode at first and then to step to the acceleration mode (iv). Therefore more than half of the total beam study period assigned for the RCS so far was used for the beam storage mode.

The capability of the injection and extraction beam dumps are $4 \mathrm{~kW}$ in one-hour average, and most of the beam used for the RCS beam tuning and study was dumped there.
In the following sections, beam commissioning results obtained in each operation mode are described.

\section{TUNING OF THE INJECTION BEAM}

Figure 2 shows a schematic view of the RCS injection section. For the beam injection, two types of orbit-bump systems [23] are prepared. One is the shift-bump system (four horizontal pulse dipole magnets; SB1-4) to make a horizontal orbit offset at the injection point, which produces a flattop field during the injection. The other is the paint-bump system (four horizontal and two vertical pulse dipole magnets; PBH1-4 and PBV1-2) prepared for the transverse beam painting. In the initial beam tuning phase, a simple center injection with no beam painting scheme was employed with only SB1-4, where the chopped pulses from the linac were multiturn injected at the center of the RCS beam ellipse in the phase space. As SB1-4 with a large gap have a large fringe field and are very closely installed near to each other and also to the quadrupole magnets (QFL and QDL), the magnetic interference and its resultant imbalance of their field integrals are not negligible. Since SB1-4 are connected in series and cannot be adjusted independently, they can be an additional source of the closed orbit distortion during the injection period. Therefore their gap adjustments were carefully performed in advance based on their field measurements coupled with the quadrupole magnet in order to fulfill a "closed" injection-orbit bump [24]. As mentioned in the next section, the closed orbit distortion caused by SB1-4 was confirmed to be negligible in the beam storage mode.

In the injection area, three types of carbon stripper foils [25] are installed. The first one is the main stripper foil utilized for the charge-exchange injection for $\mathrm{H}^{-}$from the linac. The second and third ones are used for charge exchanging $\mathrm{H}^{0}$ and $\mathrm{H}^{-}$unstripped on the first foil and delivering them to the injection beam dump. The injection

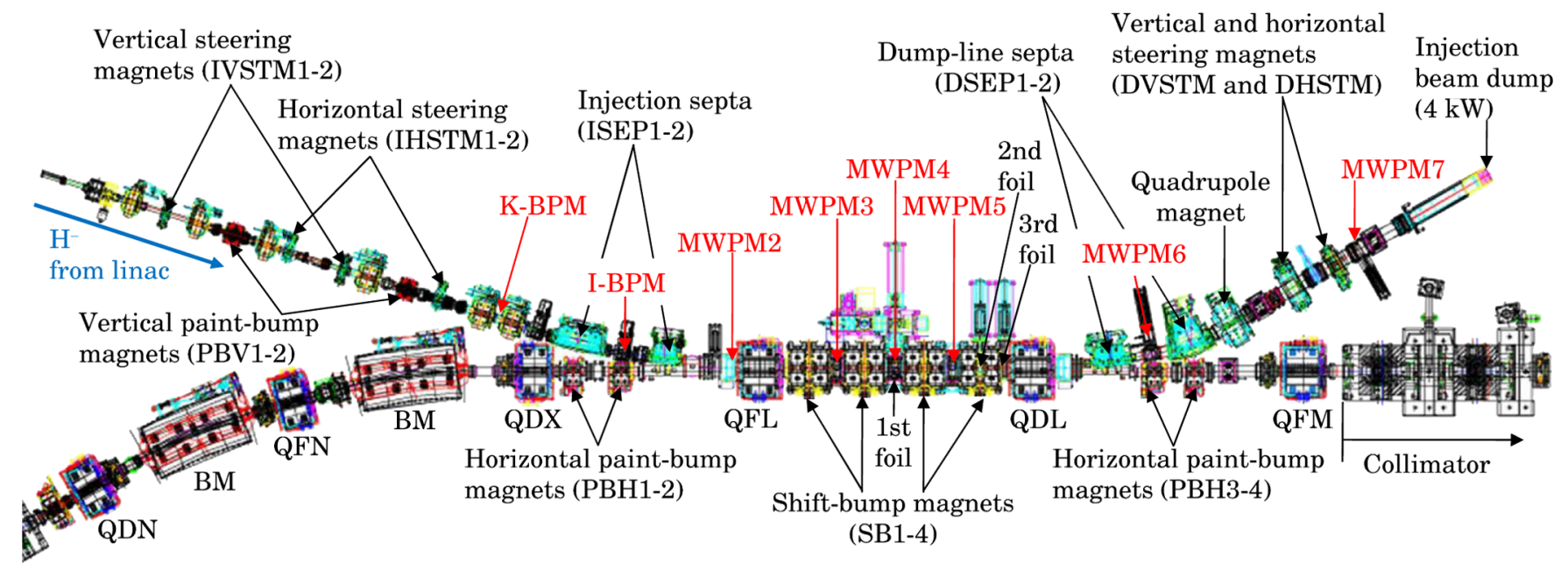

FIG. 2. (Color) Schematic view of the RCS injection area, where the red characters correspond to the beam diagnostics devices defined in Table II. 

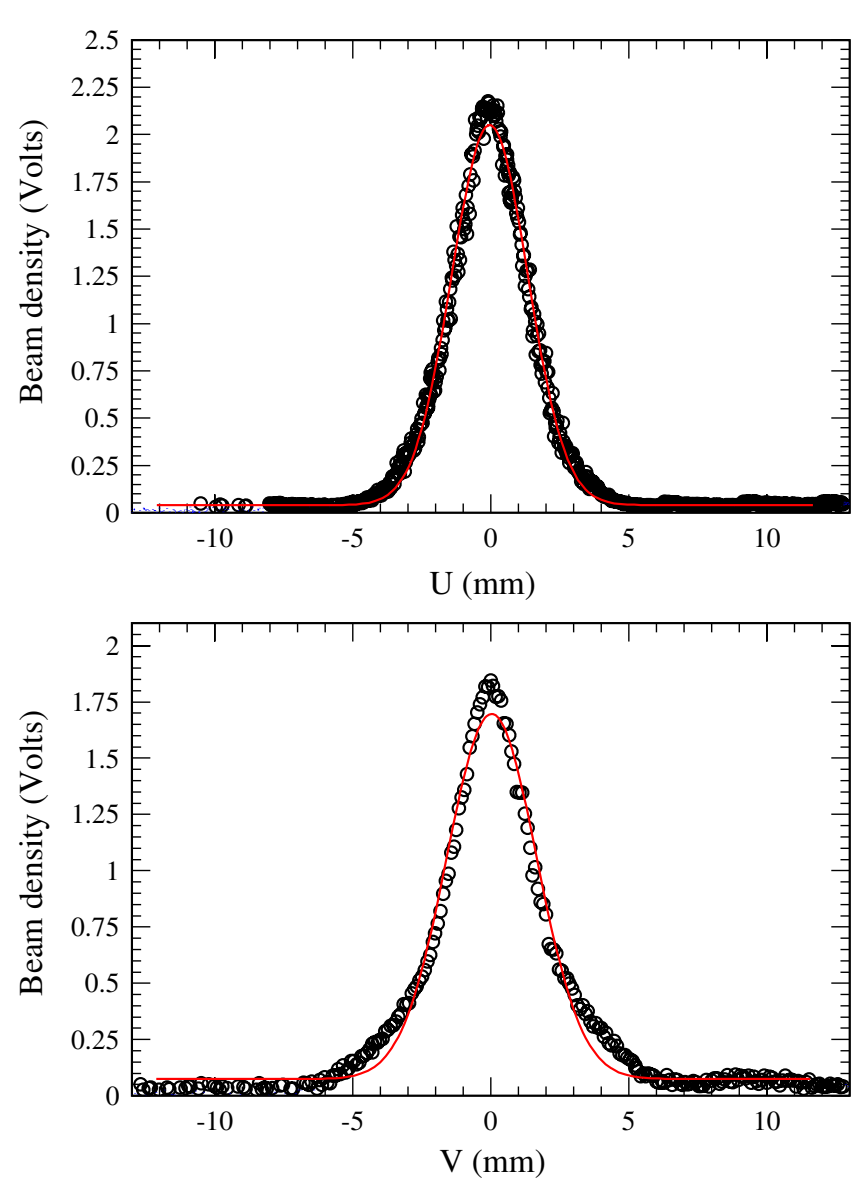

FIG. 3. (Color) Transverse profiles of the injection beam measured by MWPM4 with two wire sensor frames, where the wires are put parallel to the $\mathrm{U}$ and $\mathrm{V}$ axes 17.7 degrees rotated from the horizontal and vertical axes on the transverse two-dimensional plane. Therefore the beam profiles in this figure are given as the projections to the $\mathrm{U}$ and $\mathrm{V}$ axes. Solid red curves are Gaussian fitting results.

beam was basically tuned up in the injection beam dump mode, where the first foil was removed and the linac beam was directly driven to the injection beam bump.

The injection and dump-line orbits were mainly adjusted using MWPM2-7. Figure 3 shows beam profiles measured with MWPM4 installed closely to the first foil. The beam centers and widths on MWPM's were defined by Gaussian fitting. After the L-3BT beam tuning was fixed, we first roughly adjusted the injection and dump-line orbits, and then performed a series of orbit response matrix measurements such as MWPM3-5 vs injection septa (ISEP1-2 [26,27]), MWPM6-7 vs dump-line septa (DSEP1-2 $[26,27])$ and MWPM4 vs SB1-4 for the horizontal plane, and MWPM3-5 vs vertical steering magnets (IVSTM1-2) for the vertical plane. The measured response matrices were almost consistent with the calculated ones. The injection and dump-line orbits were successfully controlled by the measured response matrices or in combination with the calculated ones.

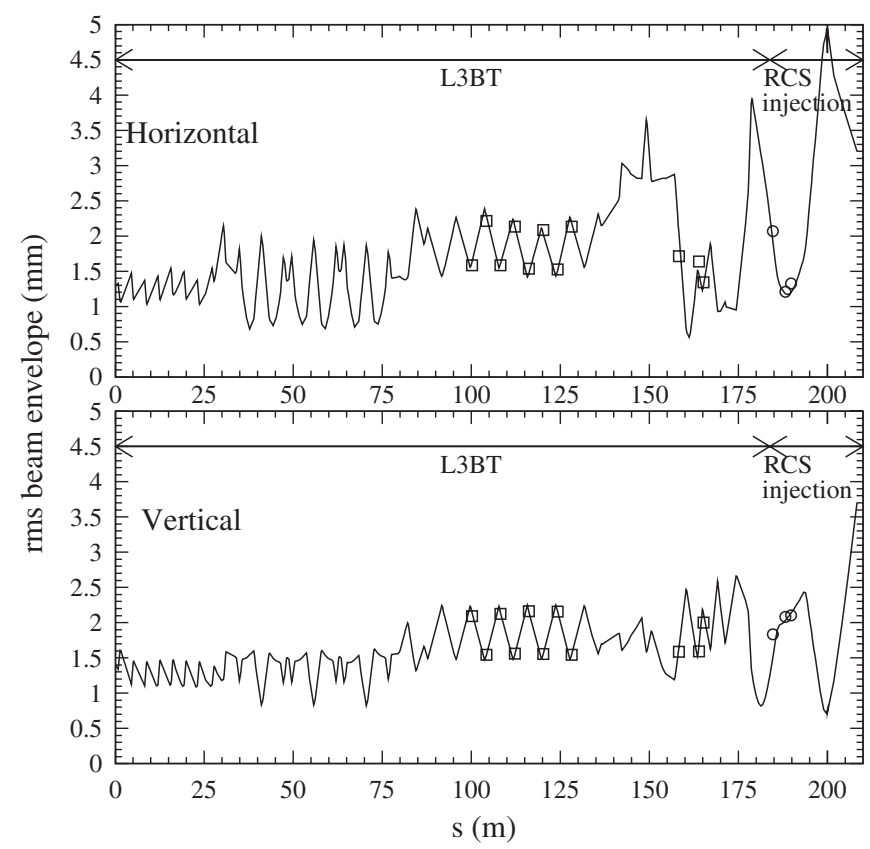

FIG. 4. Comparisons of the beam profile widths on MWPM2-4 with the rms beam envelopes (solid curves) obtained in the transverse matching at wire scanner profile monitors located at L-3BT, where the upper and lower ones are for the horizontal and vertical planes, and circles and squares show the rms beamwidths measured on MWPM2-4 and L-3BT wire scanners.

Figure 4 shows rms envelopes of the injection beam obtained in the transverse matching procedure with wire scanner profile monitors located at L-3BT [22]. Solid curves in the figure are the result from a model-based fitting to the L-3BT profiles (11 squares in Fig. 4) with the transverse Twiss functions and beam emittances at the L-3BT entrance as free parameters. The rms beamwidths measured on MWPM's (three circles in Fig. 4) located at the RCS injection section were nearly consistent with the beam envelopes simply extrapolated from L-3BT based on the quadrupole set-points.

\section{BEAM STORAGE MODE}

A series of basic parameter tuning measurements was performed in the beam storage mode. This operation mode does not involve the acceleration process and, consequently, it is more straightforward to capture a basic behavior of the beam. The essence of our beam tuning scenario is to clearly understand the basic performance of the RCS in the beam storage mode and to define the initial acceleration patterns based on the parameters tuned up in this mode.

At first, in this mode, the ring dipole magnetic field strength was fitted to the injection beam momentum by looking at horizontal closed orbit displacements at the dispersive arc sections, where no rf was employed, and beam position data for the first 50 turns $(0.1 \mathrm{~ms})$ for which 
the beam still kept a bunch structure were utilized to compute the closed orbit. Next the revolution frequency was measured, and then the beam was captured in the rf stationary bucket, where the rf frequency and phase offsets were chosen to minimize longitudinal dipole oscillations. After that, closed orbit corrections, and then a series of optics measurements and correction were performed. Finally, the injection orbit was precisely adjusted to the ring closed orbit so as to minimize betatron oscillations. Several beam studies, such as investigations on the effects of leakage fields from the injection and extraction lines and betatron resonances, were also performed in this mode.

\section{A. Closed orbit correction}

Figure 5 shows closed orbit distortions (COD) measured with 54 sets of ring-BPM's before and after the COD correction performed with 52 sets of steering magnets. During $20 \mathrm{~ms}$ from injection through extraction, 20 successive COD measurements at regular 1-ms intervals (0.1 ms data taking and $0.9 \mathrm{~ms}$ intermission) were processed. While the COD was observed to be around $10 \mathrm{~mm}$ at maximum (black squares), it was well corrected to be less than $2 \mathrm{~mm}$ (red circles) within a few iterations by using a singular value decomposition solver for a model-based response matrix of closed orbit positions on ring-BPM's vs kick angles of the steering magnets.

Blue asterisks in the figure show a COD difference when the injection-orbit bump is on and off. The COD variation caused by SB1-4 was within $\pm 0.5 \mathrm{~mm}$, and it was con-

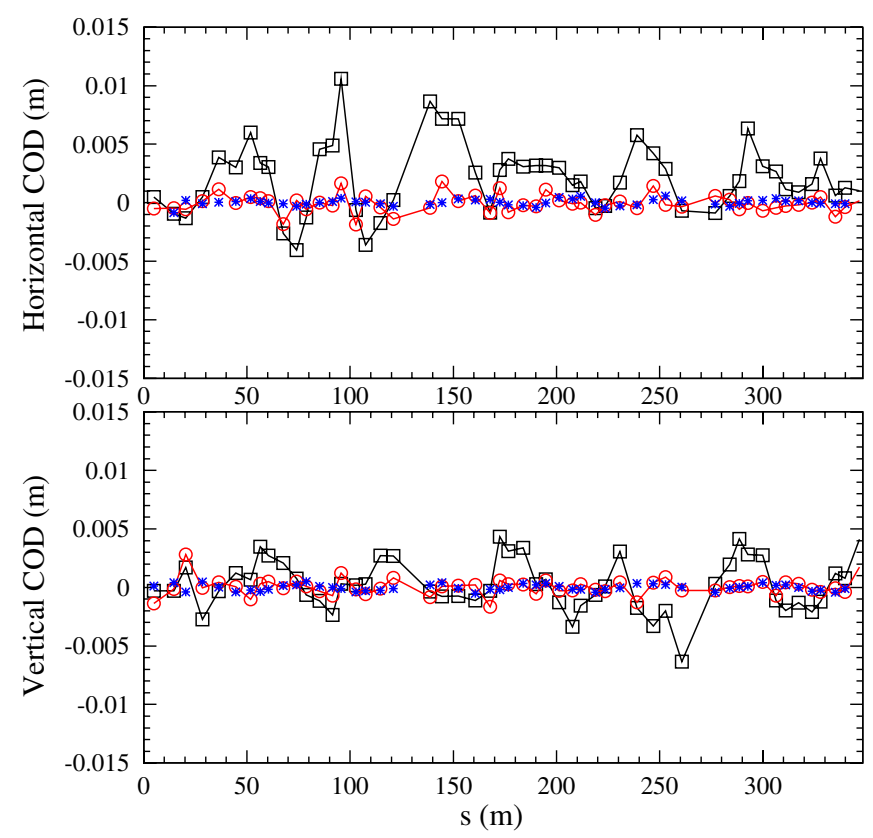

FIG. 5. (Color) Horizontal (upper) and vertical (lower) COD measured on ring-BPM's in the beam storage mode before (black squares) and after (red circles) the COD correction, where blue asterisks show the COD difference when the injection-orbit bump is turned on and off. firmed in this measurement that a closed injection-orbit bump was successfully formed, as mentioned in the last section.

\section{B. Optics measurement and its correction}

After fixing the closed orbit, a series of optics measurements was performed. Figure 6 shows dispersion and
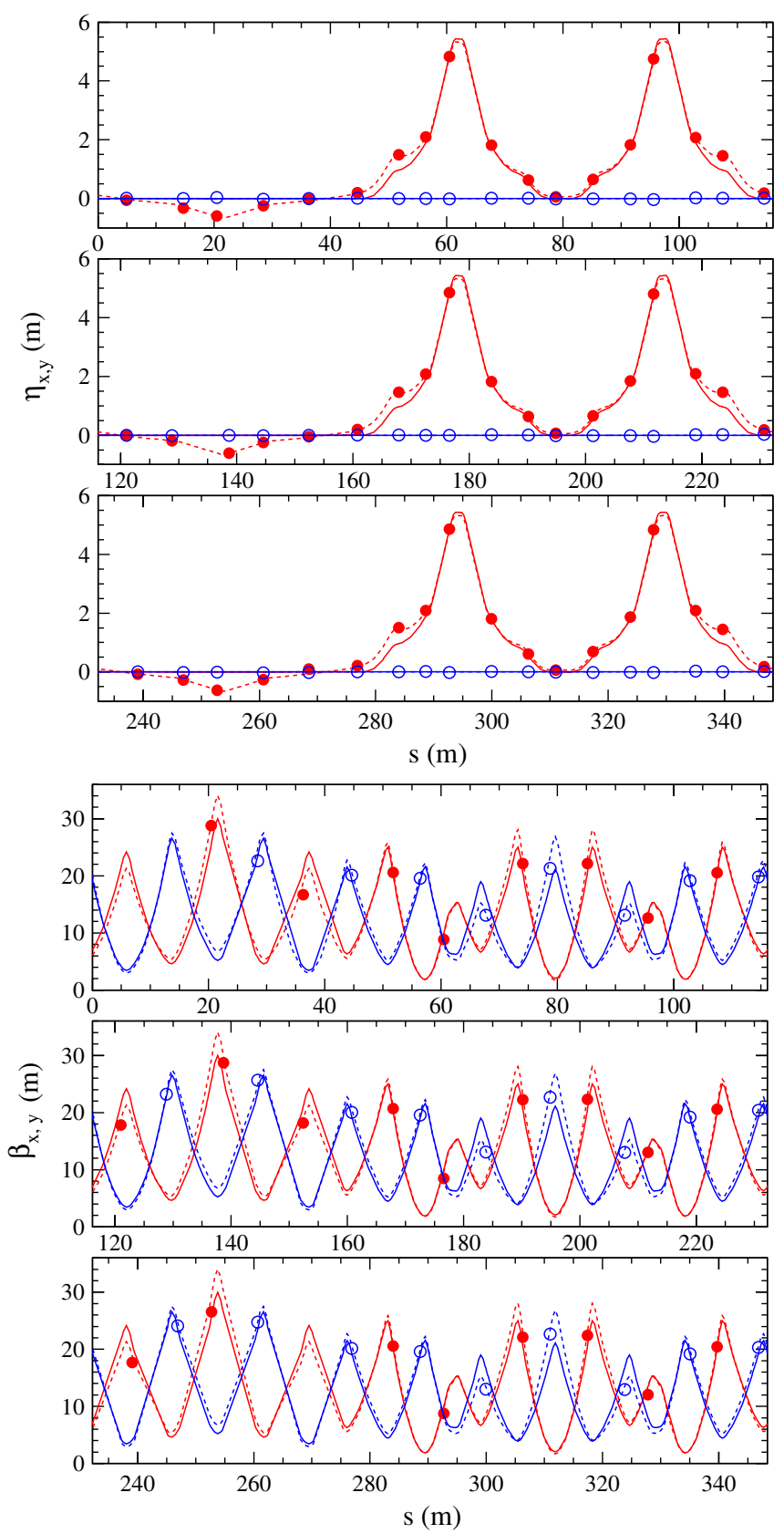

FIG. 6. (Color) Dispersion (upper) and betatron amplitude (lower) functions observed in each first measurement in the beam storage mode, where closed red circles are the horizontal ones, open blue circles the vertical ones, dashed curves show the reconstructed optical functions in our calculation model, and solid curves the design. 
betatron amplitude functions obtained in each first measurement in the beam storage mode.

The dispersion function was evaluated by looking at an rf frequency dependence (within $\pm 0.6 \%$ in momentum) of the closed orbit, where the rf frequency was adiabatically varied with a linear ramp to each final value making use of the first $10 \mathrm{~ms}$ in order to avoid longitudinal smearing by a dipole oscillation. In this way, closed orbit data after $10 \mathrm{~ms}$ where the rf frequency was set to be constant were utilized to estimate the dispersion function.

On the other hand, the betatron amplitude function was evaluated using a COD formula. The closed orbit shift on the $i$ th ring-BPM induced by a kick angle of the $j$ th steering magnet is expressed as the following:

$$
\begin{aligned}
\delta z_{\mathrm{co}}^{j}\left(s_{i}\right)= & \frac{1}{2 \sin \pi \nu_{z}} \delta \theta_{z}^{j} \sqrt{\beta_{z}\left(s_{i}\right) \beta_{z}\left(s_{j}\right)} \\
& \times \cos \left[\pi \nu_{z}+\left|\psi_{z}\left(s_{i}\right)-\psi_{z}\left(s_{j}\right)\right|\right],
\end{aligned}
$$

where $s$ is the arclength measured along the reference closed orbit, $z$ stands for either horizontal $x$ or vertical $y$ coordinate in the reference-orbit curvilinear coordinate system $(x, y, s), \nu$ is the betatron tune, $\beta$ the betatron amplitude function, $\delta \theta$ the steering kick angles, and $\psi$ the betatron phase advance. Most of the ring-BPM's are installed inside of the steering magnets and the betatron phase difference of each pair can be presumed to be negligible. Accordingly, the betatron amplitude function $\beta_{z}\left(s_{j}\right)$ at each steering magnet is given as a function of observable closed orbit response $\delta z_{\mathrm{co}}^{j}\left(s_{j}\right) / \delta \theta_{z}^{j}$ and $\nu_{z}$ from Eq. (1):

$$
\beta_{z}\left(s_{j}\right)=2 \frac{\sin \pi \nu_{z}}{\cos \pi \nu_{z}}\left(\frac{\delta z_{\mathrm{co}}^{j}\left(s_{j}\right)}{\delta \theta_{z}^{j}}\right)
$$

While the closed orbit and betatron tune were directly measured with a sufficient accuracy, there was a large ambiguity in the steering kick angles. Our thin steering magnets with large gaps (330-410 mm gap/100 mm long core) cause a large fringe field around their magnet edge. Therefore a part of the fringe field is absorbed by nearby components such as a quadrupole magnet. For this concern, several field measurements and simulations of steerers coupled with a quadrupole or dipole magnet were performed. As the measured and simulated results were in good agreement, we performed a series of field simulations for all the steering magnets and applied the simulated ones to the kick angle corrections. Figure 7 shows the simulated field reduction rates, which are 10\%-30\% depending on the ferromagnetic material environment near to the steerers [28].

While the optical functions, betatron tunes, and dispersion and betatron amplitude functions obtained in each first measurement were slightly shifted from the design, they

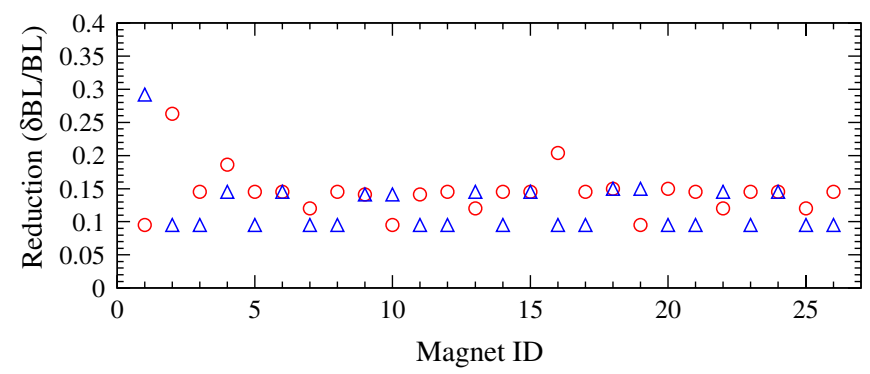

FIG. 7. (Color) Calculated field reduction rates of the horizontal (red circles) and vertical (blue triangles) steering magnets caused by the magnetic interference.

were well reconstructed in a unified view in our SAD [29]based calculation model, as shown with dashed curves in Fig. 6. It means a series of optics measurements including the kick angle corrections for the steering magnets is successfully performed. This model fitting in the leastsquares method fed offsets for seven families of quadrupole magnets to compensate the discrepancy of the design and measured optical functions, namely, a difference between the quadrupole field sets which gave the design optical system (solid curves in Fig. 6) and reproduced the measured ones (dashed curves in Fig. 6). As shown in Fig. 8, the optical system was successfully corrected by the offsets with no iteration. While the applied offsets slightly change for different operating tunes, we may be able to find a better parametric formalism, which gives parameters common for a wide range of tunes, by a systematic survey in the betatron tune diagram.

\section{Chromaticity measurement and its correction}

After establishing the basic optical system, we carried out a chromaticity measurement and its correction, as shown in Fig. 9. The chromaticity was estimated by looking at an $\mathrm{rf}$ frequency dependence of the betatron tune in a similar way as the dispersion measurement. The natural chromaticity was measured to be $-10.3 /-7.2$ for the horizontal/vertical planes. The RCS magnets have a large gap and a nonlinear behavior of their edge field plays a significant role. Our calculation model in which such a field nonlinearity based on their field measurement is included [12,13] gives a consistent magnitude of $-10.4 /-6.8$, while it is $-8.5 /-8.1$ if using a simple model with only linear property. The chromaticity correction was also successfully performed with field strengths of three families of sextupole magnets determined by the calculation model.

\section{Injection error correction}

Finally in a series of basic parameter tuning, a residual injection error, namely, a betatron oscillation caused by an offset relative to the closed orbit, was corrected. In this tuning, a single short pulse injection, where the beam from 

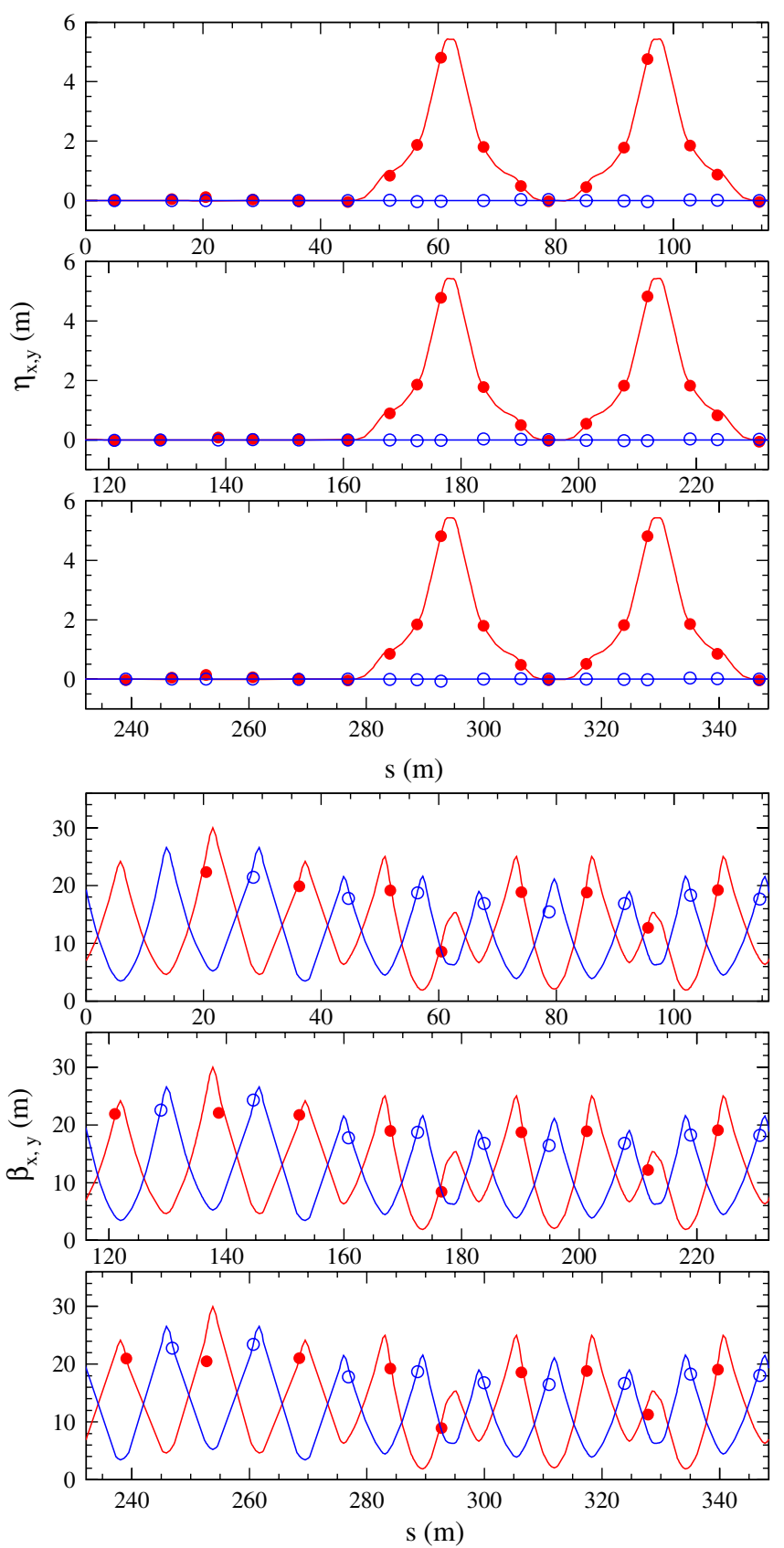

FIG. 8. (Color) Corrected dispersion (upper) and betatron amplitude (lower) functions observed in the beam storage mode, where closed red circles are the horizontal ones, open blue circles the vertical ones, and solid curves the design.

the ion source was chopped to make a single turn (typically $25 \mathrm{~mA}$ peak and $560 \mathrm{~ns}$ long), was employed to clearly capture a betatron oscillation. In this tuning procedure, turn-by-turn beam position data on two sets of ringBPM's displaced by a drift space were utilized to identify the phase space coordinates $\left(x, x^{\prime}=\frac{d x}{d s}\right)$ and $\left(y, y^{\prime}=\frac{d y}{d s}\right)$ of the injection beam at the first stripper foil [30]. The phase space coordinates measured at the BPM pair were transferred to the injection point with a transfer matrix obtained

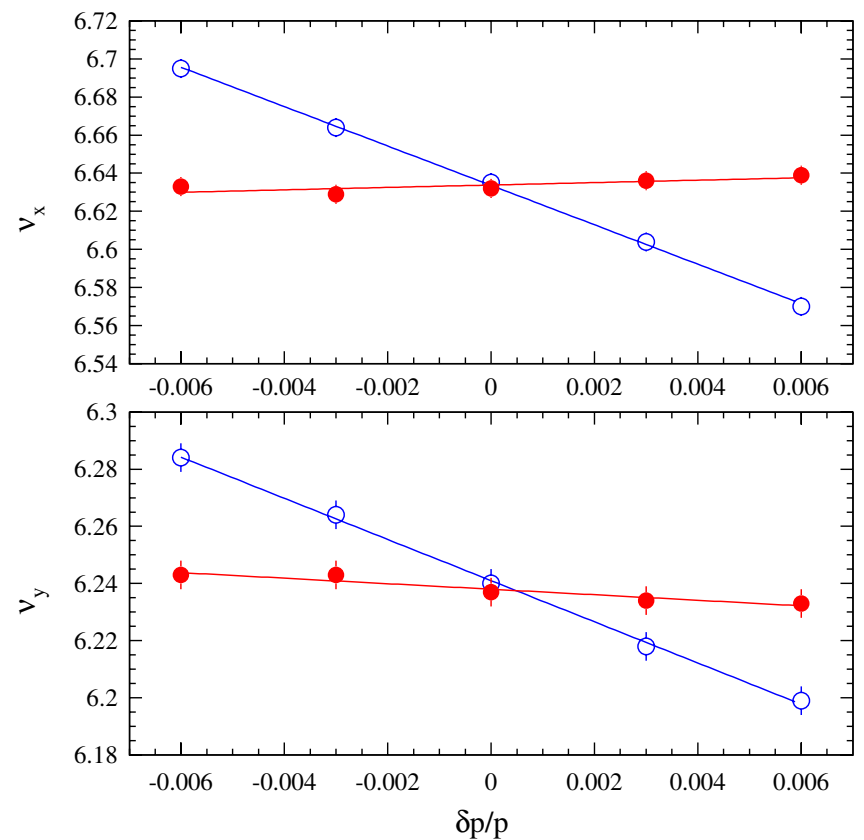

FIG. 9. (Color) Horizontal (upper) and vertical (lower) betatron tunes as a function of the momentum offset measured in the beam storage mode before (open blue circles) and after (closed red circles) the chromaticity correction.

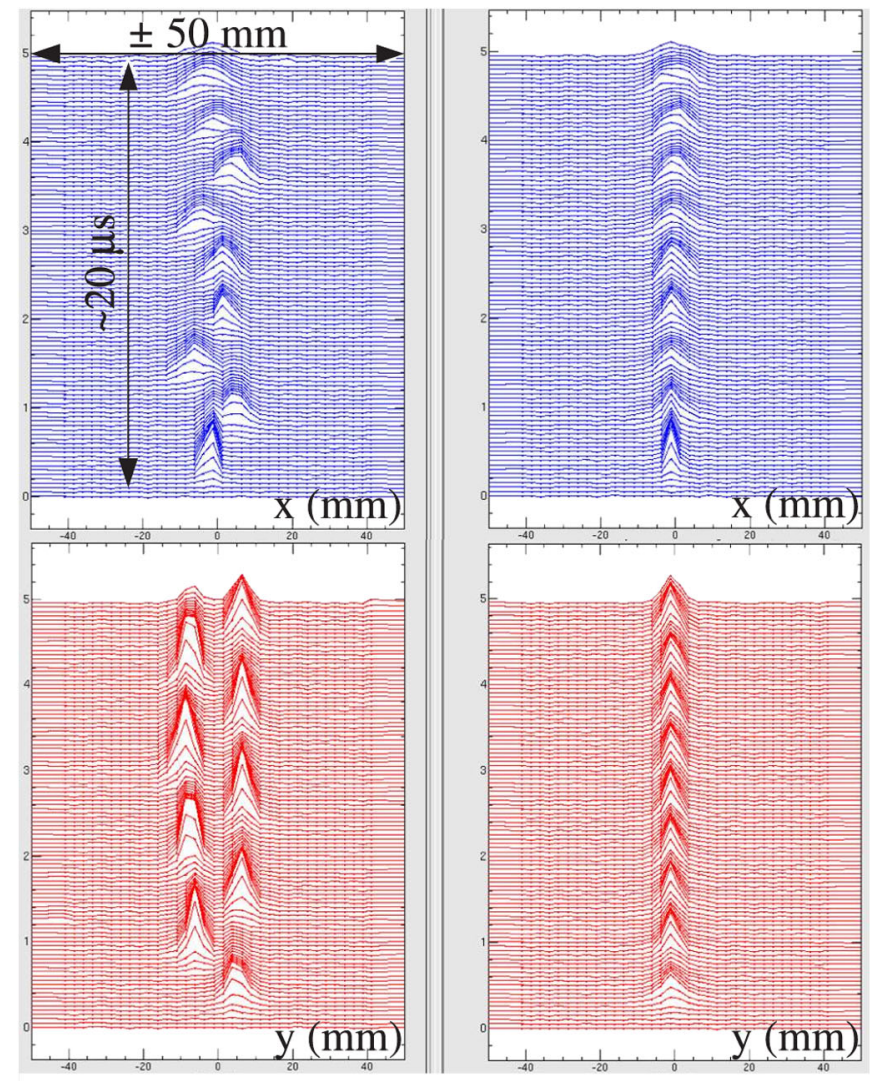

FIG. 10. (Color) Horizontal (upper) and vertical (lower) beam profile mountain views for the first nine turns measured with IPM before (left) and after (right) the injection error correction. 
by fitting the measured optics with our calculation model. The measured injection errors were well corrected within a few iterations using model-based or measured orbit response matrices for kick angle variations of the injectionline magnets. Figure 10 shows beam profile mountain views measured by IPM before and after the correction. It clearly shows the betatron oscillations diminish by the correction. Because the injection beam passes through two ring quadrupole magnets (QFL and QDL) as shown in Fig. 2, this tuning procedure is required whenever they are changed such as when the operating tune is altered. Therefore such a quick tuning technique with a minimum of iterations is essential to perform our beam studies efficiently.

\section{E. Linear coupling}

The linear coupling was examined by observing the horizontal (vertical) closed orbit displacement for $1 \mathrm{mrad}$ vertical (horizontal) single kick at 26 different points with the steering magnets, as shown in Fig. 11. The kicks produced around $10 \mathrm{~mm}$ COD's in the corresponding plane, for which the orbit leaks to the horizontal and vertical planes were measured to be around 0.12 and $0.09 \mathrm{~mm}$ in rms, where the contributions from the rotation errors of BPM's and steering magnets were estimated to be less than $0.004 \mathrm{~mm}$. The observed small horizontal-vertical coupling reflects distributed errors such as quadrupole rotations and residual COD's at the sextupole magnets.
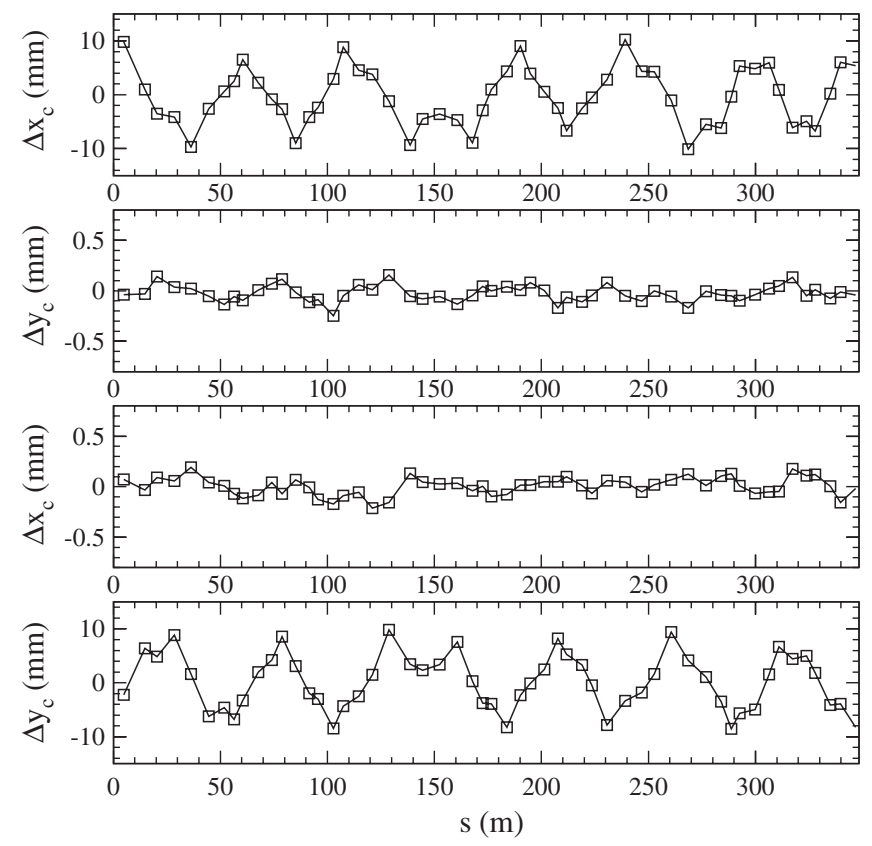

FIG. 11. Typical example of the horizontal and vertical closed orbit displacements $\left(\Delta x_{c}\right.$ and $\left.\Delta y_{c}\right)$ for $1 \mathrm{mrad}$ horizontal single kick (upper two plots) and for $1 \mathrm{mrad}$ vertical single kick (lower two plots), measured in the beam storage mode.

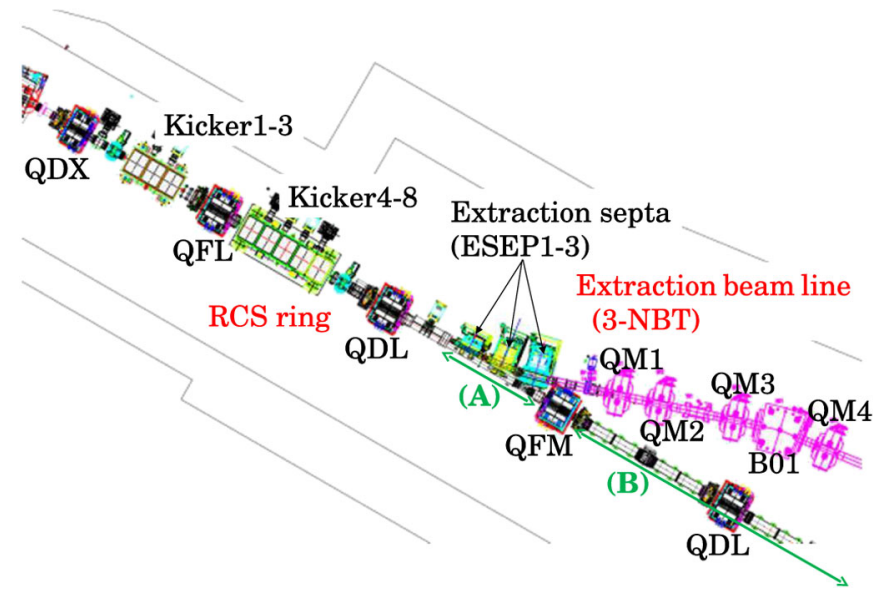

FIG. 12. (Color) Schematic view of the RCS extraction area.

\section{F. Static leakage fields from the extraction beam line}

As shown in Fig. 19 later, the COD before the correction observed in the $3 \mathrm{GeV}$ acceleration mode gradually diminishes during acceleration, which implies that static magnetic fields from DC-excited magnets located at the extraction beam line leak to the ring. For this concern, we quantitatively investigated the leakage field magnitude in the $181 \mathrm{MeV}$ beam storage mode with different current sets of the extraction DC magnets without beam extraction for the case that the current set was shifted from that corresponding to the circulating beam energy.

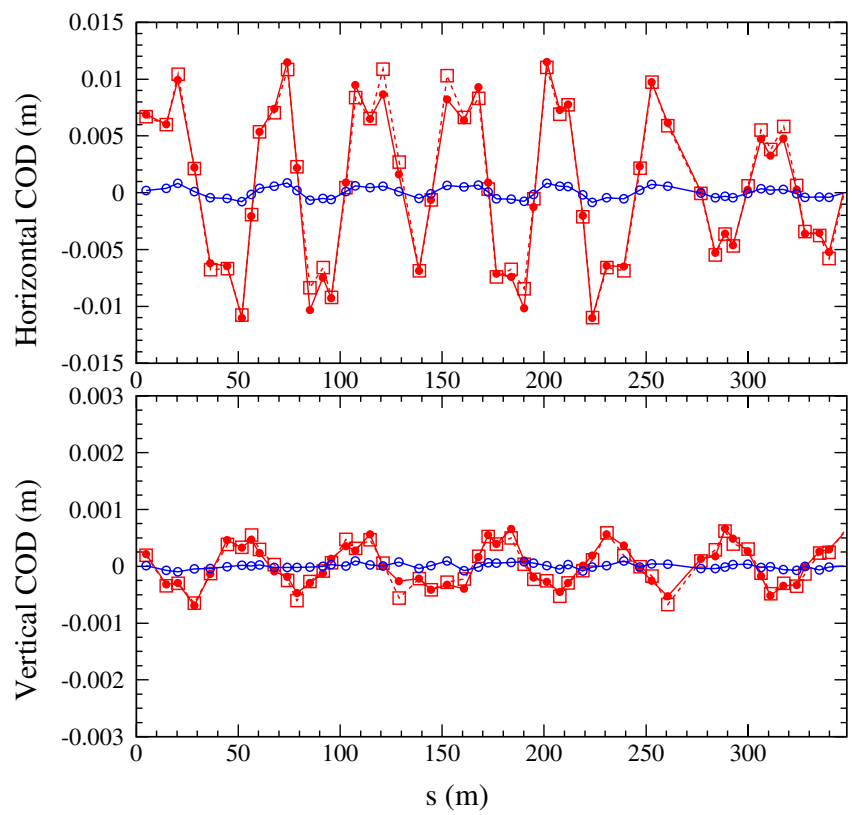

FIG. 13. (Color) Horizontal (upper) and vertical (lower) COD caused by the leakage field from ESEP excited at $181 \mathrm{MeV}$ (open blue circles) or at $3 \mathrm{GeV}$ (closed red circles) measured in the $181 \mathrm{MeV}$ beam storage mode, in which open red squares show the COD obtained in the model fitting for the closed red circle data. 
As shown in Fig. 12, three sets of septum magnets (ESEP1-3 [26,27]) are installed in the extraction area. Figure 13 shows the COD caused by a leakage field from ESEP excited at $181 \mathrm{MeV}$ (open blue circles) or at $3 \mathrm{GeV}$ (closed red circles), which was derived from a difference of COD's observed when turning ESEP on (at $181 \mathrm{MeV}$ or at $3 \mathrm{GeV}$ ) and off. The leakage field was more significant when ESEP was excited at $3 \mathrm{GeV}$, which made a COD of 12 and $0.8 \mathrm{~mm}$ for the horizontal and vertical planes. By fitting the measured COD distribution with our calculation model assuming field error sources at the extraction area, as shown with open red squares in Fig. 13, the magnitude of the leakage field was evaluated to be $1.4 \times 10^{-3} / 0.9 \times$ $10^{-4}$ Tesla $\cdot \mathrm{m}$ for normal/skew dipole components. Similar results were also obtained for a leakage field from DC magnets located at 3-NBT, especially the first dipole magnet (B01 in Fig. 12); $1.8 \times 10^{-3} / 1.6 \times$ $10^{-4}$ Tesla $\cdot \mathrm{m}$. Figure 14 shows the leakage field distributions measured on the surface of the vacuum ducts along the ring [31]. The leakage field distribution from ESEP has a characteristic peak structure and it seems that their static field leaks to the ring directly from the coils through the magnetic shield gaps. On the other hand, the leakage field from $\mathrm{B} 01$ which is located around $3.5 \mathrm{~m}$ away from the ring is locally not so large, but it is distributed in the broader area. The residual fields, which the measured field distributions made inside of the vacuum ducts, were evaluated considering the shielding effects; $1.7 \times 10^{-3} \mathrm{Tesla} \cdot \mathrm{m}$ from ESEP and $1.9 \times 10^{-3}$ Tesla $\cdot \mathrm{m}$ from B01 (normal

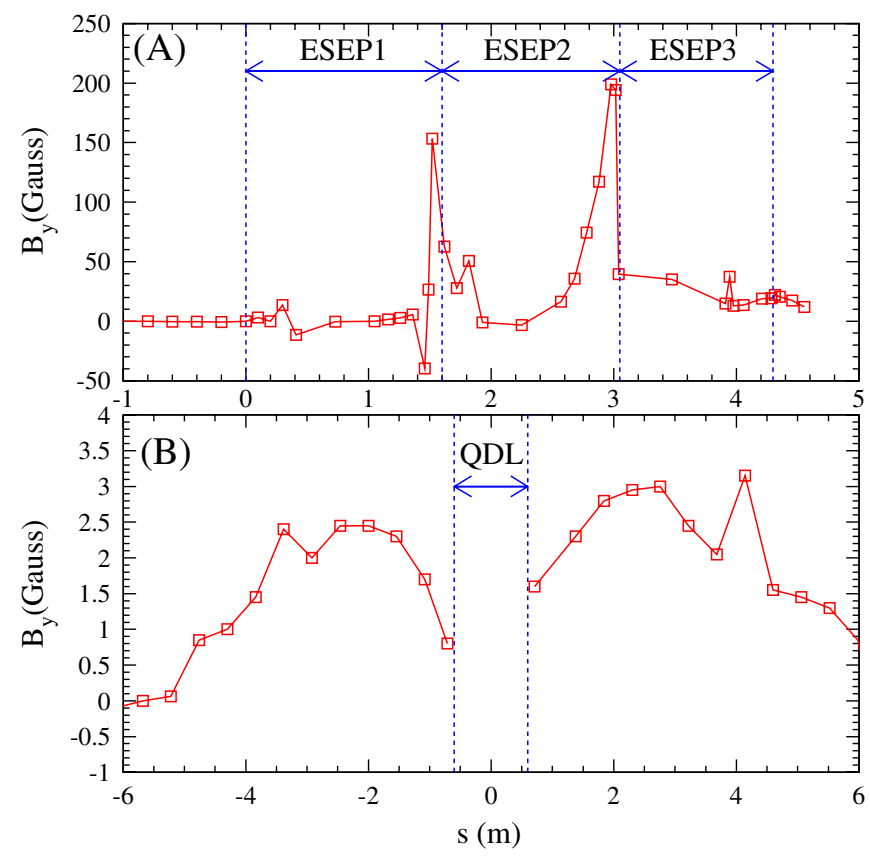

FIG. 14. (Color) Leakage field distributions from ESEP (A) and B01 (B) excited at $3 \mathrm{GeV}$ measured on the surface of the vacuum ducts along the ring using a Hall probe, where the measured areas of (A) and (B) are figured by green arrows in Fig. 12. dipole component), which are in good agreement with the estimations from the COD analyses. Also as to DSEP1-2, similar measurements and analyses were performed. The DC magnets located at the injection section are normally excited at $181 \mathrm{MeV}$. Thus, their leakage field was more than one order of magnitude smaller than those of the extraction DC magnets.

Since COD caused by such a leakage field can be sufficiently corrected by steering magnets, COD itself will not lead to serious problems. However, in general, it includes higher-order field components such as a quadrupole component, and can distort the superperiodicity of an accelerator, additionally exciting several nonstructure betatron resonances. In fact, the betatron tunes slightly changed for different current sets of the extraction DC magnets. For example, the betatron tune $\left(\nu_{x}, \nu_{y}\right)$ was measured to be $(6.635,6.237)$ at the $181 \mathrm{MeV}$ current set and $(6.624,6.242)$ at $3 \mathrm{GeV}$ with a resolution of 0.0013 , where $\mathrm{COD}$, which varied depending on the current set, was corrected with different steering field sets for both cases to minimize a tune change coming from a COD difference. By fitting such a tune change with our calculation model, the integrated magnitude of the resulting normal quadrupole field component was evaluated to be 0.02 Tesla.

\section{G. Betatron tune survey}

In order to investigate the effects of the leakage fields on the circulating beam in more detail and also for clear understanding of the stability and flexibility of the betatron tune space, we performed betatron tune surveys in the beam storage mode, and compared them with singleparticle tracking simulations.

\section{Measurement}

Figure 15 shows beam survival as a function of the betatron tune, measured with DCCT, where the tunes were varied along arrows shown in Fig. 17. In Fig. 15, blue squares correspond to the case that the extraction DC magnets are excited at $181 \mathrm{MeV}$, and red circles show the case of excitation at $3 \mathrm{GeV}$ for which the leakage fields are more significant. While the COD varied depending on the current set of the extraction DC magnets, it was corrected to be less than $2 \mathrm{~mm}$ with different steering field sets for both cases. As shown in the figure, several betatron resonances were observed; half integer resonances $2 \nu_{x, y}=13$, a linear coupling sum resonance $\nu_{x}+\nu_{y}=13$, a thirdorder structure resonance $\nu_{x}-2 \nu_{y}=-6$ mainly from the chromaticity correction magnets and a sextupole field component intrinsic in the main dipole magnets, and so on. This figure shows the leakage fields enhance the half integer and linear coupling resonances, which means there exists a significant skew quadrupole field component as well as the normal one in the leakage fields. The skew quadrupole component magnitude is supposed to be one order of magnitude smaller than the normal one consider- 

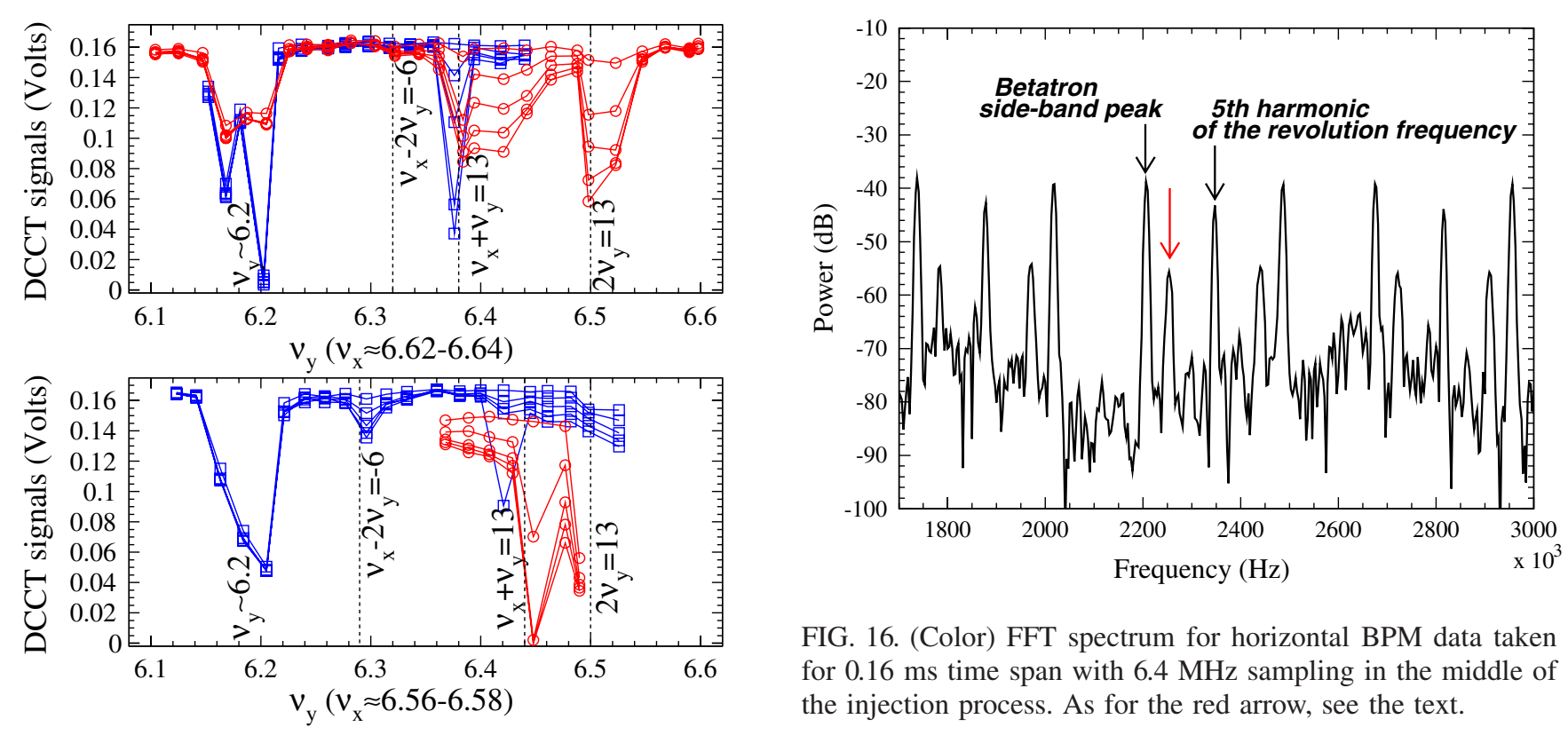

FIG. 16. (Color) FFT spectrum for horizontal BPM data taken for $0.16 \mathrm{~ms}$ time span with $6.4 \mathrm{MHz}$ sampling in the middle of the injection process. As for the red arrow, see the text.

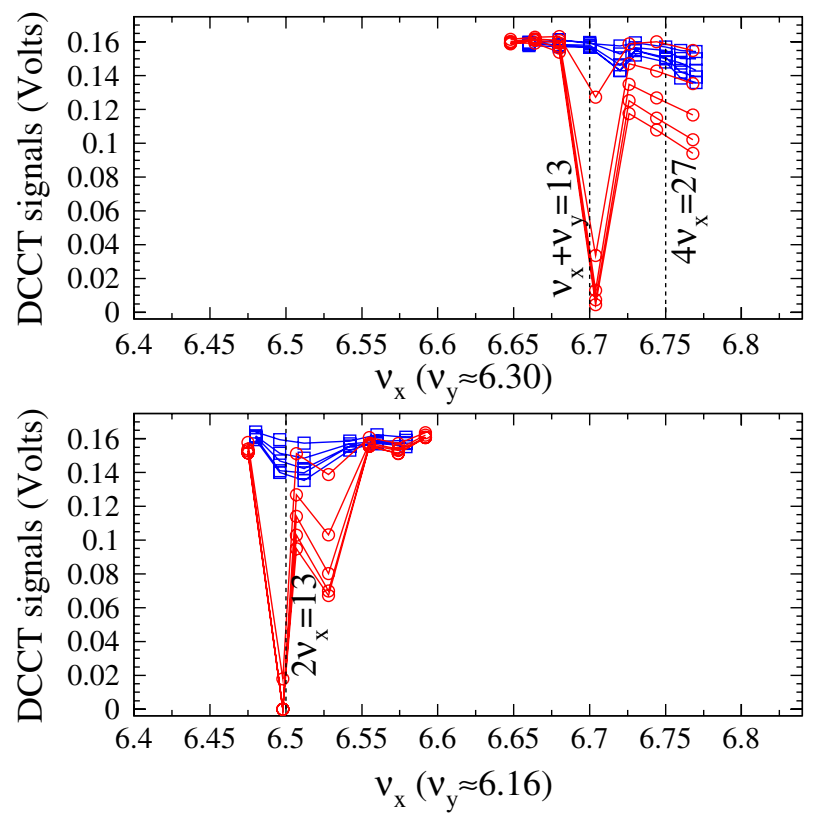

FIG. 15. (Color) Beam survival at 1, 5, 10, 15, and $19 \mathrm{~ms}$ as a function of the betatron tune, measured with DCCT in the $181 \mathrm{MeV}$ beam storage mode, where the extraction DC magnets were excited at $3 \mathrm{GeV}$ (red circles) or at $181 \mathrm{MeV}$ (blue squares).

ing the ratio of the normal and skew dipole field components evaluated in the previous subsection.

In these measurements, a low-intensity $\left(4.2 \times 10^{11}\right.$ particles per bunch) and small-emittance (around $40 \pi \mathrm{mm} \mathrm{mrad}$ ) beam formed by the center injection was utilized. Therefore, higher-order resonances, such as $\nu_{x}-2 \nu_{y}=-6$ and $4 \nu_{x}=27$, tend to be $\operatorname{dim}$ in this measurement. A wider beam and/or higher-intensity beam enhancing the space-charge effect will give a different situation.

As shown in the two upper plots of Fig. 15, a large beam loss was observed along $\nu_{y}=6.2$. A similar beam loss was found also on $\nu_{x}=6.2$ which was rather critical. In a fastFourier-transform (FFT) spectrum for BPM data, another peak at the corresponding frequency was always observed with a significant intensity for both the horizontal and vertical planes in addition to multiples of the revolutionfrequency (around $469 \mathrm{kHz}$ ) and betatron sideband peaks, as shown with a red arrow in Fig. 16. It appeared only around a time range of the injection period and thereafter disappeared. In fact, these beam losses were very fast and almost all the particles were lost in the first $1 \mathrm{~ms}$ not depending on the beam intensity, especially for $\nu_{x}=6.2$. It was also confirmed in the $3 \mathrm{GeV}$ acceleration mode that such a fast beam loss did not take place at the middle and late stage of the acceleration period $(20 \mathrm{~ms})$, where the quadrupole field patterns were manipulated so that the tune crossed the line at the middle of the acceleration cycle. These situations say that they are not connected to spacecharge induced effects, beam instabilities, and also higherorder nonlinear resonances such as fifth-order parametric resonance. One possibility is a ripple current of the SB1-4 power supply. SB1-4 are used to make an injection-orbit bump during around $1 \mathrm{~ms}$ (typically $0.6 \mathrm{~ms}$ flattop for the injection and $0.5 \mathrm{~ms}$ fall time at present). The FFT spectrum of the search-coil signal for the injection-bump field gives a significant peak at $96 \mathrm{kHz}$, which just corresponds to the anomalous sideband peak in Fig. 16. The observed ripple is equivalent to the second harmonic of the switching frequency $(48 \mathrm{kHz})$ of the insulated gate bipolar transistor in the SB1-4 power supply, which may excite a forced beam oscillation. These resonance lines impose a strong limitation on the betatron tunability. The identification of the origin and a countermeasure for that are one of our important issues at present. 

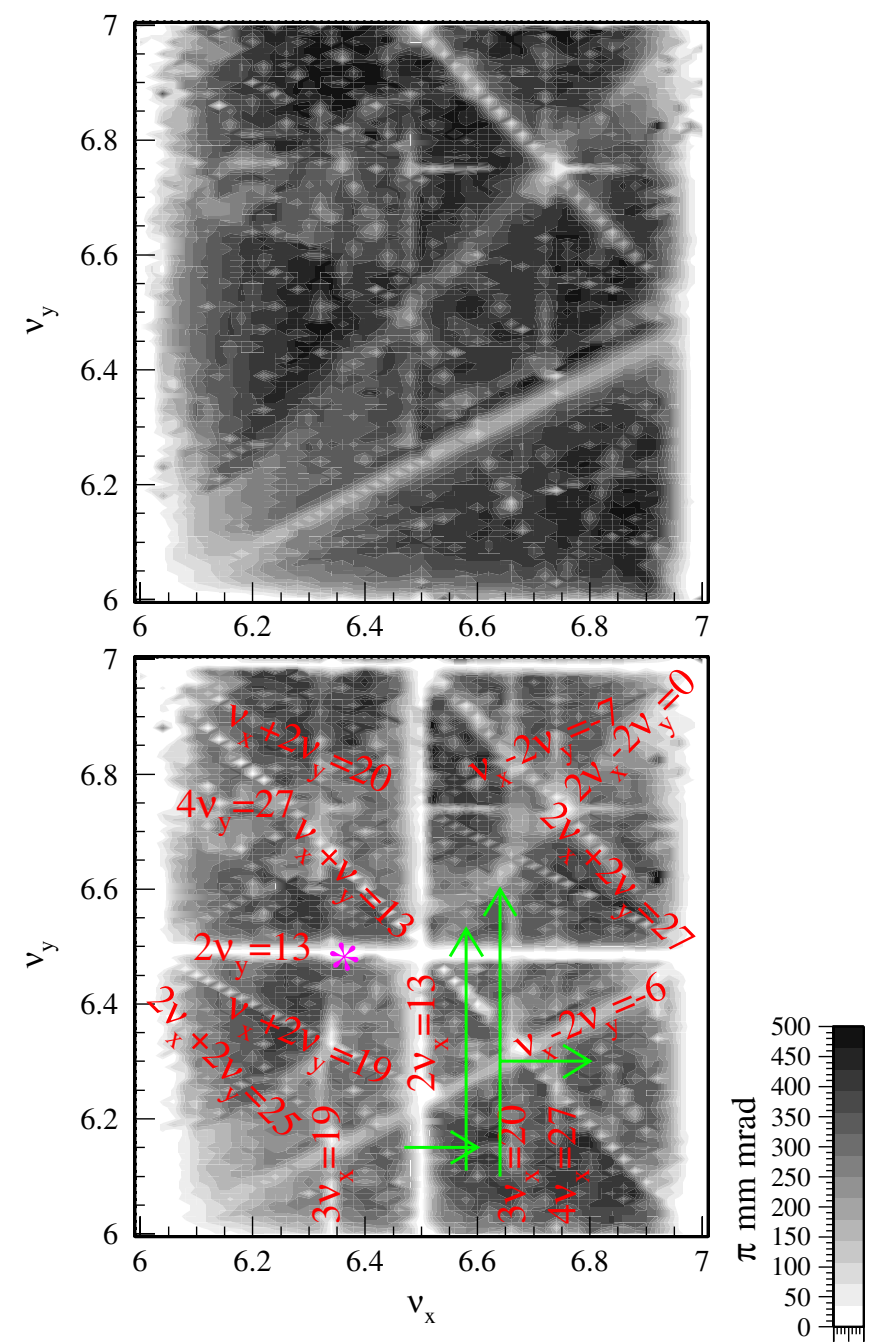

FIG. 17. (Color) Dynamic aperture maps obtained from singleparticle tracking simulations without (upper) and with (lower) the leakage fields from the extraction DC magnets.

\section{Single-particle tracking simulation}

Figure 17 shows dynamic aperture maps obtained from single-particle tracking simulations assuming the beam storage mode at $181 \mathrm{MeV}$ without and with the leakage fields estimated above. Possible intrinsic nonlinear fields (chromatic correction sextupole fields and multipole field components for all the ring magnets based on their field measurement) were also included together with measured field and alignment errors [12,13]. In the simulations, actual physical apertures were set for all the magnets, and for a beam particle with the initial condition of $\epsilon_{x}=$ $\epsilon_{y}, x=\sqrt{\epsilon_{x} / \gamma_{x}}, x^{\prime}=0, y=\sqrt{\epsilon_{y} / \gamma_{y}}, y^{\prime}=0, \phi=0$, and $\delta p / p=0$, we examined a maximum value of $\epsilon_{x}=\epsilon_{y}$ for which the beam survived within the physical apertures up to 5000 turns, where $\left(x, x^{\prime}\right)$ and $\left(y, y^{\prime}\right)$ are the horizontal and vertical phase space coordinates, $(\phi, \delta p / p)$ the longitudinal phase and the momentum offset, $\epsilon$ the beam emit- tance, and $\alpha, \beta$, and $\gamma \equiv\left(1+\alpha^{2}\right) / \beta$ are the Twiss parameters. These simulations show a similar situation as the measured one that the leakage fields excite half integer resonances $2 \nu_{x, y}=13$ and a linear coupling sum resonance $\nu_{x}+\nu_{y}=13$. In these maps, various higher-order resonances are found as well as the identified ones in the above tune surveys. Third- and fourth-order structure resonances can be identified in both maps; $\nu_{x}-2 \nu_{y}=-6$ from the chromatic correction magnets and a sextupole field component in the main dipole magnets, and $2 \nu_{x}-$ $2 \nu_{y}=0,2 \nu_{x}+2 \nu_{y}=27$, and $4 \nu_{x, y}=27$ induced by an octupole field component in the fringe fields of the main magnets and by the second-order effect of the sextupole fields. These simulations also show that the leakage fields excite various third- and fourth-order nonstructure resonances through a distortion of the superperiodicity caused by their quadrupole field component; $\nu_{x}+2 \nu_{y}=19$ and 20, $\nu_{x}-2 \nu_{y}=-7,3 \nu_{x}=19$ and 20 , and $2 \nu_{x}+2 \nu_{y}=$ 25 . In order to obtain better stability and flexibility of the betatron tune space, a countermeasure against the leakage fields is essential. This is also a key issue to gain the effectiveness of the beam painting scheme especially in the transverse plane. For this concern, we plan to install additional shields to make the leakage one order of magnitude smaller than the current one.

\section{ACCELERATION MODE}

We began on a tuning for the acceleration mode with the initial acceleration patterns based on the ring parameters defined in the beam storage mode.

Also in the acceleration mode, at first, the strength at the bottom (at the injection) of the ring dipole magnetic field pattern was fitted to the injection energy in a similar way as that in the beam storage mode. Next the rf frequency pattern and the COD were corrected over the acceleration process. After that, we performed various measurements on transverse and longitudinal motions over the acceleration cycle and examined their consistency with calculations and/or those obtained in the beam storage mode. Fine-tuning was also carried out, such as a timing adjustment of extraction pulse kickers toward the two-bunch operation. Via such a beam tuning procedure, we demonstrated high beam-power operations and also tested a control of the tune variation over the acceleration process for beam loss reduction.

\section{A. Rf frequency and closed orbit corrections over the acceleration cycle}

As already mentioned, COD's from different sources, such as COD caused by static leakage fields (variable during acceleration) and COD caused by field and alignment errors (almost constant over the acceleration cycle), were separately measured in the beam storage mode. Using such data, the steering magnetic field patterns for the 


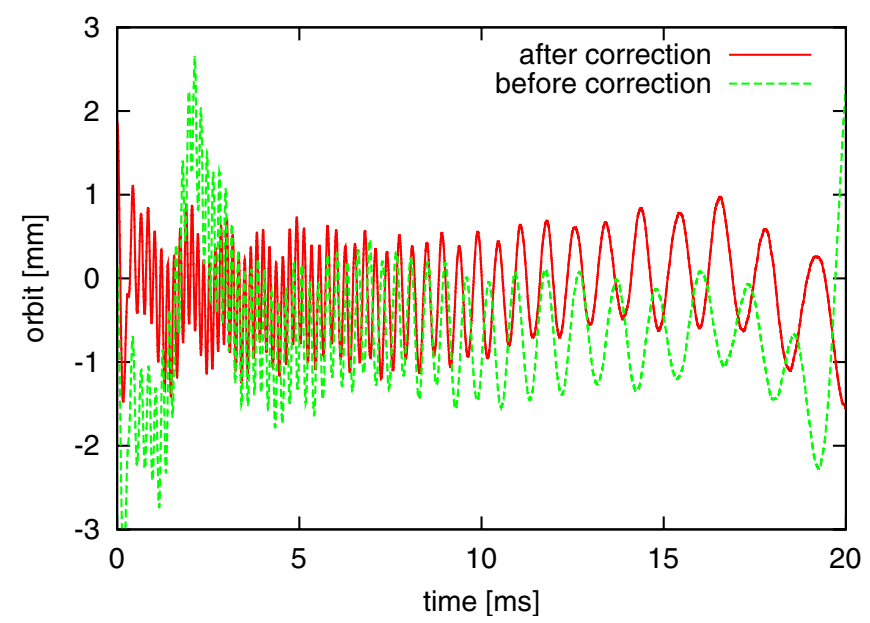

FIG. 18. (Color) Horizontal beam position data on $\Delta$ R-BPM over the acceleration process $(20 \mathrm{~ms})$ before (dashed green curve) and after (solid red curve) the rf frequency tuning.

acceleration were made in advance, with which COD over the acceleration time was roughly corrected from the beginning of the tuning in the acceleration mode. Under such a situation, the rf frequency $\left(f_{\text {rf }}\right)$ was first corrected so as to minimize the deviation of the horizontal beam position in the dispersive arc sections caused by a mismatch to the dipole field pattern, where three sets of $\triangle \mathrm{R}-\mathrm{BPM}$ prepared for the rf radial feedback system were utilized. The correction value was derived through $-f_{\text {rf }} \times$
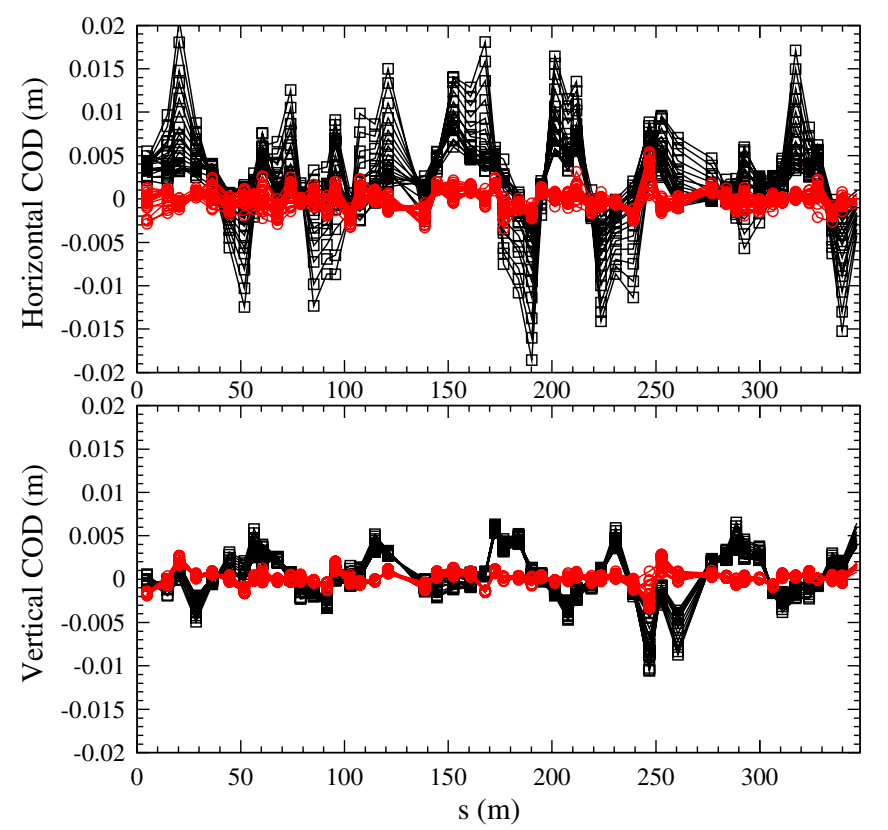

FIG. 19. (Color) Horizontal (upper) and vertical (lower) COD on ring-BPM's without (black squares) and with (red circles) the COD correction by the steering magnets, where 20 COD's are plotted for each at $1 \mathrm{~ms}$ regular intervals over the acceleration cycle $(20 \mathrm{~ms})$.

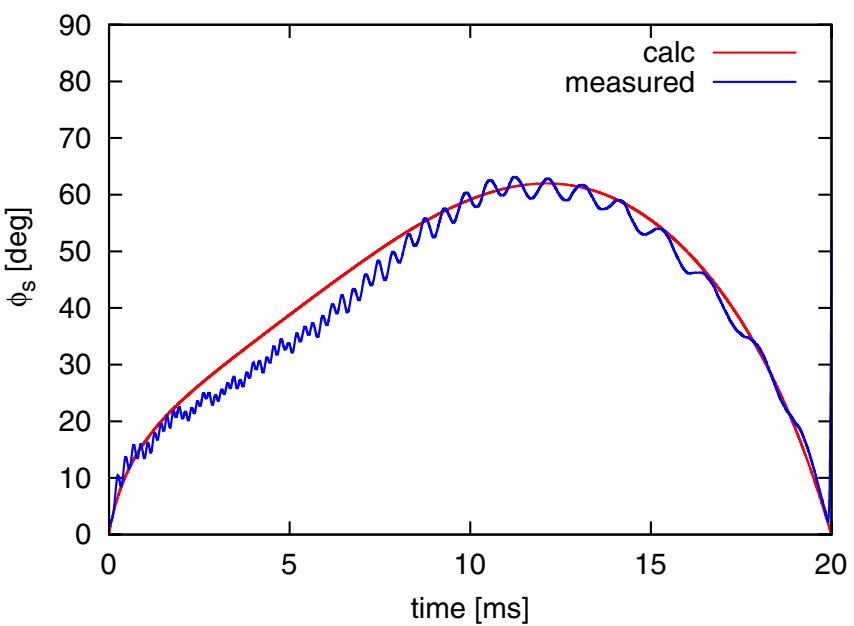

FIG. 20. (Color) Synchronous phase measured for the rf voltage shown in Fig. 21 over the acceleration time, where the blue curve is the measured one, and the red curve the calculated one assuming that the rf voltage is a pure sine wave.

(phase-slip factor $) \times(\Delta \mathrm{R} /$ dispersion $)$ using the beam position shift $(\Delta \mathrm{R})$ on $\Delta \mathrm{R}$-BPM measured over the acceleration cycle. Figure 18 shows horizontal beam position data on $\triangle \mathrm{R}-\mathrm{BPM}$ before and after the rf frequency tuning. Once the rf frequency pattern was fixed, the orbit was kept within a few mm with no online radial feedback loop through the run cycle especially thanks to the excellent stability of the ring dipole field pattern. After that, a residual COD was corrected by modifying the steering field patterns. In this way, the influences from transverse and longitudinal motions were separately well compensated. As shown with red circles in Fig. 19, the variable COD was finally corrected to within a few $\mathrm{mm}$ throughout the acceleration period over the ring.

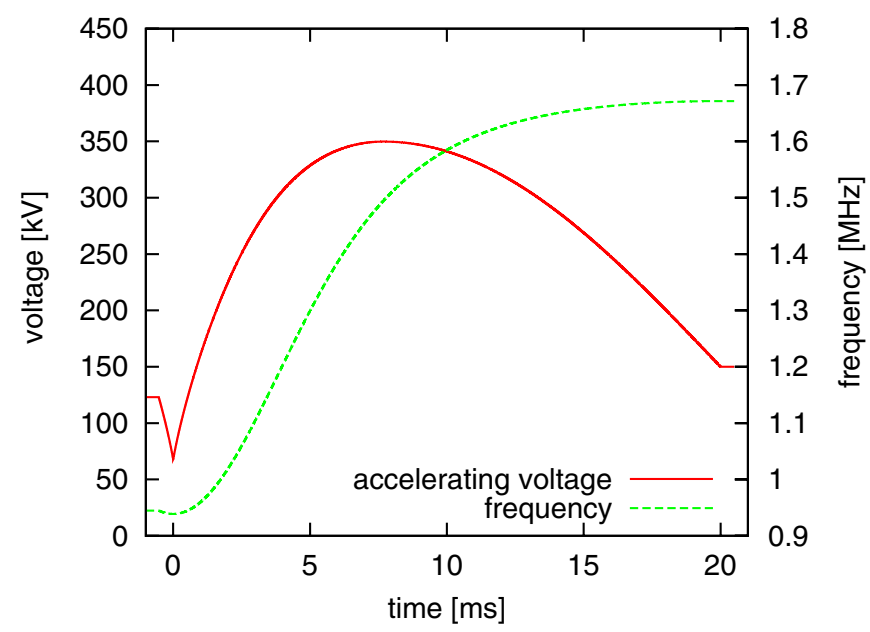

FIG. 21. (Color) Typical rf voltage pattern $(h=2)$ programmed in the $3 \mathrm{GeV}$ acceleration mode. 


\section{B. Synchronous phase measurement}

Our rf system consists of ten sets of high-gradient magnetic alloy loaded cavities (another two cavities will be installed in the near future), which can produce $41 \mathrm{kV} /$ turn per one cavity at full capability [10]. Figure 20 shows a synchronous phase measured for the rf voltage pattern $(h=2)$ shown in Fig. 21 over the acceleration period, where a blue curve shows the measured one, and a red curve the calculated one assuming that the rf voltage is a pure sine wave. The measured and calculated synchronous phases were in good agreement, which confirmed that the rf voltages set and actually felt by the beam were consistent with each other [32]. A slight difference seen from 2 to $8 \mathrm{~ms}$ comes from a third harmonic component intrinsic in the rf voltage, which is caused by the transfer function of the high power tetrodes in the final stage amplifier. Therefore the agreement of the measurement with the calculation became much better by canceling the third harmonic component by a counterphasing method, which set a phase offset for the five odd and the five even cavities at \pm 30 degrees [32].

By virtue of the excellent stability of the linac energy, the ring dipole magnetic field, and also the rf frequency and voltage, the longitudinal motion was very reproducible every pulse.

\section{Optics over the acceleration cycle}

After fixing the closed orbit for the full acceleration process, the betatron amplitude functions at injection were evaluated by observing betatron tunes vs strength of each quadrupole family. As shown in Fig. 22, this result was compared with that in the beam storage mode. They were in good agreement, which confirmed that the optical

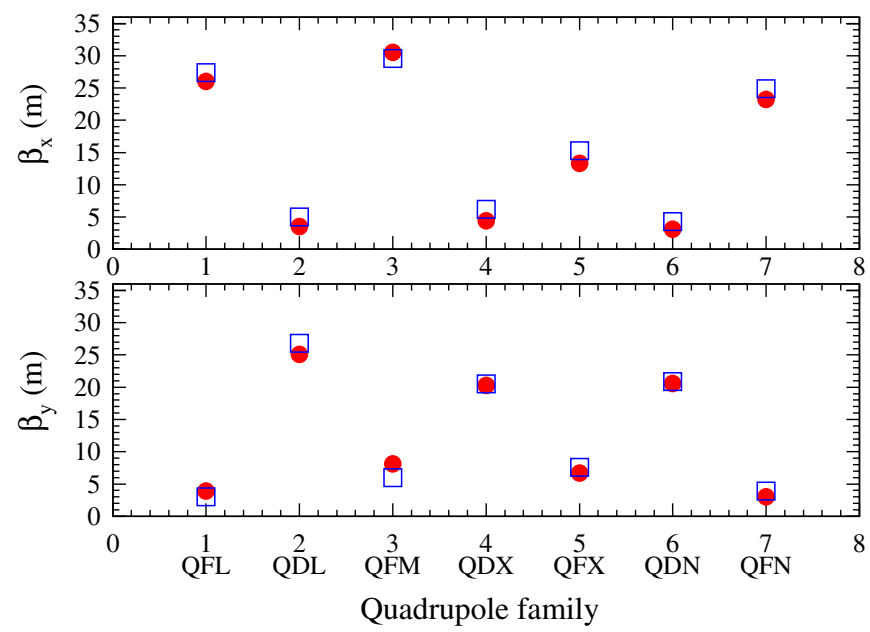

FIG. 22. (Color) Averaged horizontal (upper) and vertical (lower) betatron amplitude functions for each quadrupole family, where closed red circles are those measured at the bottom of the acceleration pattern and open blue squares are for the beam storage mode.

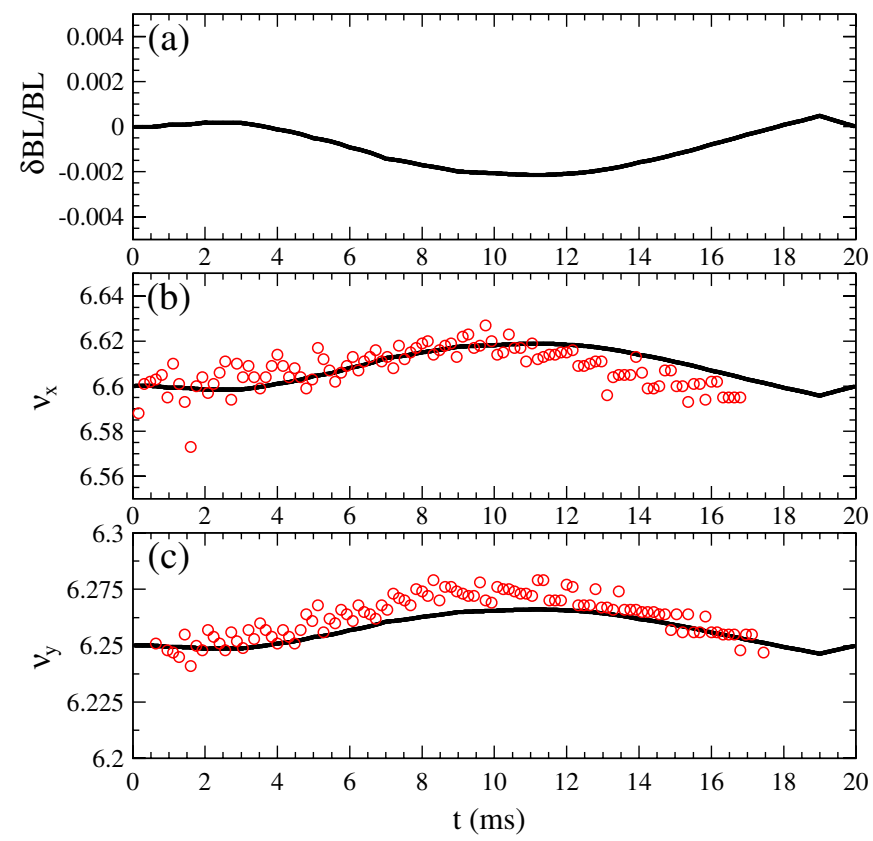

FIG. 23. (Color) (a) Deviation of the main dipole field pattern from the ideal sinusoidal field form, estimated from the rf frequency pattern. (b) Horizontal tune excursion measured over the acceleration process, where a solid curve is the calculated variation assuming the deviation of (a). (c) Vertical tune excursion.

property at the bottom of the acceleration pattern was consistent with the optics defined in the beam storage mode.

Figures 23(b) and 23(c) show betatron tune excursions measured over the acceleration process. The observed tune variations reflect a magnetic field tracking error among the main dipole and quadrupole families. The magnetic field pattern, excited with the $25 \mathrm{~Hz}$ sinusoidal current pattern, contains higher harmonic fields coming from magnetic saturation. While we aim to compensate them within a few $\times 10^{-4}$ of the $25 \mathrm{~Hz}$ fundamental field through a harmonic current control, the dipole field pattern is yet deviated from the ideal sinusoidal field form by $0.2 \%$ at maximum due to the limit of the power supply rating, as shown in Fig. 23(a), where the deviation was derived from the rf frequency pattern. Solid curves in Figs. 23(b) and 23(c) show calculated tune excursions assuming the deviation of the dipole field pattern. While the measured and calculated tune variations show a similar trend, there still remains a slight difference, which implies there exists a slight tracking error also among the quadrupole families. In order to get more precise information on the magnetic field tracking error, elaborate measurements for betatron amplitude functions as well as betatron tunes over the acceleration time are essential. Through such measurements, we will realize a closer field tracking to settle the tunes, and further aim at establishing its applied scheme, which dynamically moves the tunes over the acceleration 


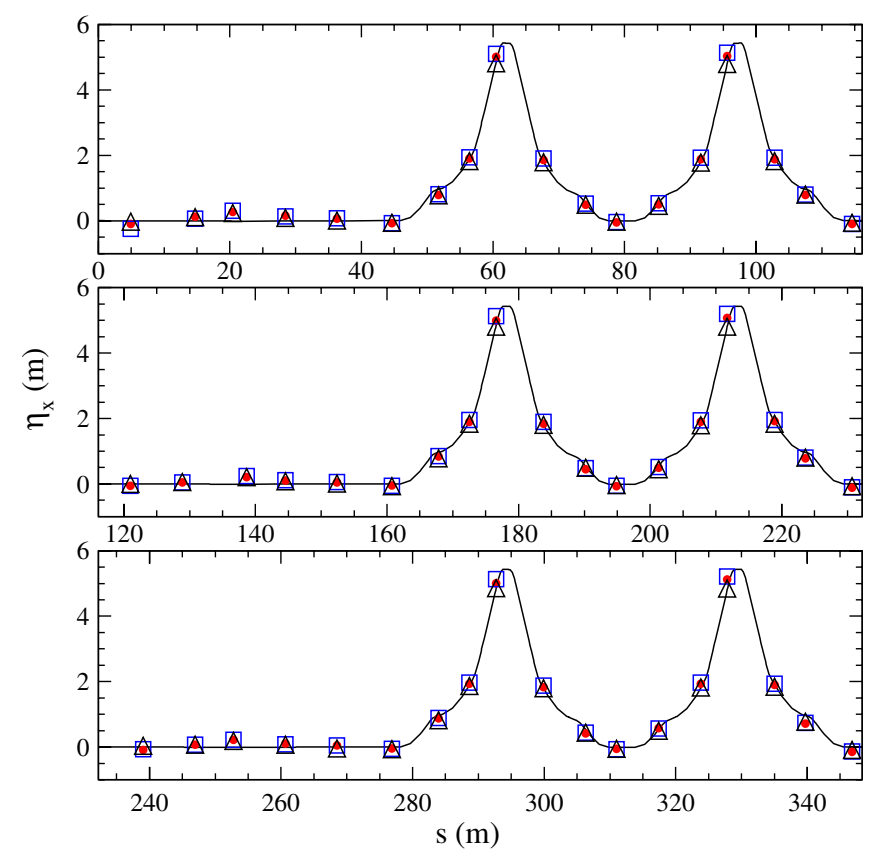

FIG. 24. (Color) Horizontal dispersions measured at 12 (closed red circles), 15 (open blue squares), and 18 (open black triangles) $\mathrm{ms}$ for the acceleration period $(20 \mathrm{~ms})$. Solid curves are the design.

cycle by manipulating DC and AC components and phase of their field pattern. Such a dynamical tune control will be very useful to manage beam losses especially for higher power operations [33], as mentioned later.

Figure 24 shows dispersion functions observed at 12, 15 and $18 \mathrm{~ms}$ for the acceleration period $(20 \mathrm{~ms})$. They were consistent with the dispersion in the beam storage mode, namely, with the design. The chromaticity was also measured over the acceleration time. At present, the chromaticity is corrected by sextupoles at the injection energy using DC power supplies. Therefore the chromaticity correction gradually fades out during acceleration. The measured result indicates such a situation.

Through such a series of measurements, it was confirmed that the optical system over the acceleration process adequately kept the condition determined in the beam storage mode.

\section{Timing adjustment for the extraction kickers}

As shown in Fig. 12, the beam extraction is performed with eight sets of pulse kickers [34]. One of the key issues for the beam extraction, especially for the two-bunch operation, was a ripple on the flattop wave form of the kickers. For this concern, several measurements and a timing adjustment for the kickers were performed [35] before two-bunch demonstrations that are mentioned in the next subsection. Figure 25(a) shows a flattop wave form of the kickers derived from the beam position shift on downstream 3-NBT beam position monitors. The kicker

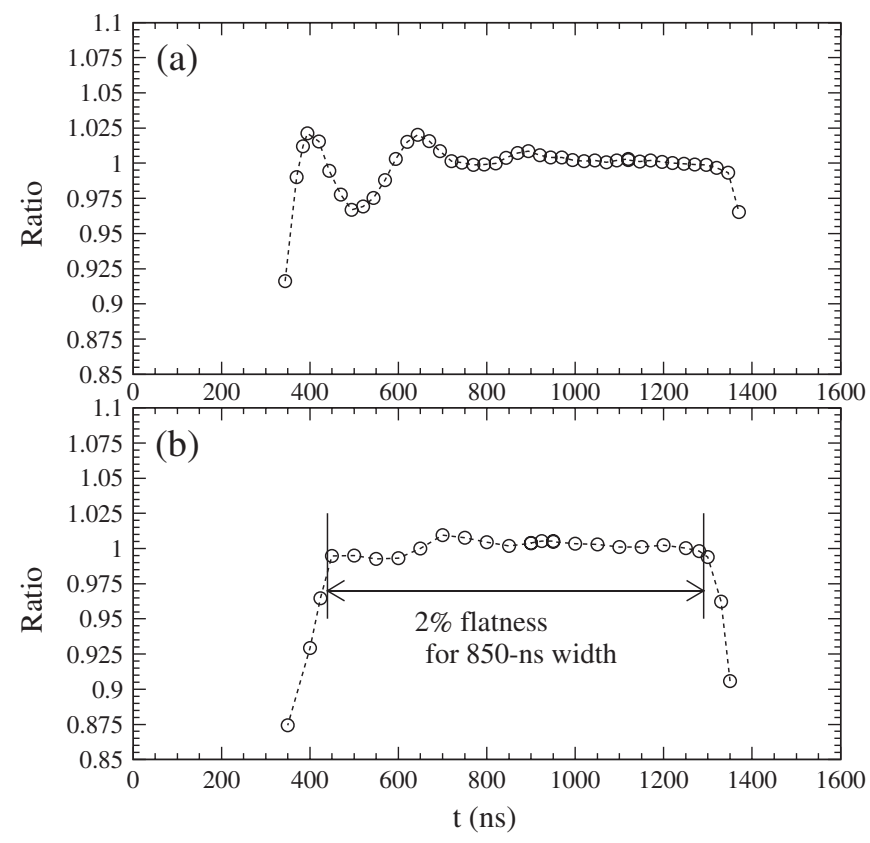

FIG. 25. Flattop wave forms of the extraction kickers before (a) and after (b) the relative timing adjustment among the kickers.

timing events can be shifted in time relative to the beam pulse. In this measurement, all the kickers were triggered with the same timing, and a range of their setting was scanned for a short beam bunch with around 30-ns length at the extraction time to obtain a fine structure of the flattop. This result shows a $6 \%$ ripple (full width) at the early region of the flattop. This observed ripple corresponds to a 16-mm beam position shift at the exit of ESEP. This effect on the beam will lead to an emittance growth and possibly cause a beam loss at the extraction. While the beam extraction for the one-bunch operation was performed utilizing the flatter region near the end of the kicker pulse, such a method cannot be applied for the twobunch operation due to the limited flattop length. For this concern, a finer adjustment of the relative timing among the kickers was carried out. Figure 25(b) shows an improved result after introducing 130-ns timing offset between the four odd and the four even kickers, where the time offset corresponds to the distance of peak and valley of the original ripple. As shown in the figure, the ripple was successfully compensated in this way, and the flatness was improved to $2 \%$ for an 850 -ns wide flattop region. This satisfies the criterion required for the final design (2\% flatness for an 841-ns flattop width).

\section{E. High power demonstration}

Via the initial beam tuning procedure mentioned above, we performed a high beam-power demonstration at $25-\mathrm{Hz}$ repetition, in which a consecutive $52 \mathrm{~kW}$ output was successfully achieved for four minutes up to the limit of the beam dump capacity ( $4 \mathrm{~kW}$ in one-hour average); 
$4.3 \times 10^{12}$ protons per bunch in the one-bunch operation using a linac beam with $23 \mathrm{~mA}$ peak, $0.12 \mathrm{~ms}$ long, and $26 \%$ chopper beam-on duty factor. This demonstration was carried out with no beam painting process. The beam intensity loss was measured to be $6.5 \%$, appearing only around the injection energy. The estimated loss corresponds to $200 \mathrm{~W}$ in power.

In addition, we demonstrated the two-bunch operation via the fine timing adjustment for the kickers mentioned above, and successfully achieved acceleration and extraction of $1.1 \times 10^{13}$ particles per pulse using a linac beam with $23 \mathrm{~mA}$ peak, $0.12 \mathrm{~ms}$ long, and $66 \%$ chopper beam-on duty factor. No significant beam loss was observed at the extraction and the beam intensity loss was similar with $6.5 \%$ only around the injection energy. While this demonstration was performed with no repetition (namely, the beam-on-demand operation), the output beam power would correspond to $130 \mathrm{~kW}$ if we were running the RCS with this intensity at $25 \mathrm{~Hz}$. This is the maximum instantaneous output achieved so far at the RCS (by June 2008).

During these demonstrations, no trouble at all, such as beam instabilities, unexpected beam losses or degradations of vacuum, was observed. Most of the lost particles around the injection energy were well localized on the ring collimators with 4-kW capacity. This was confirmed through the beam loss monitor signals during operation and the residual radiation survey performed after the demonstration.

\section{F. Tune manipulation during acceleration}

The beam intensity loss observed for $130 \mathrm{~kW}$-equivalent intensity beam (around $5 \times 10^{12}$ particles per bunch) is now improved to $2.5 \%$ by modifying the tune variation in the acceleration process as well as the betatron tune at injection. In this case, the betatron tune at injection was set at $(6.35,6.47)$ very close to the half integer line of $2 \nu_{y}=$ 13, which is marked with an asterisk in Fig. 17, in order to get enough margin for a possible space-charge tune depression in the delta surrounded by $\nu_{x}=6,2 \nu_{y}=13$, and $2 \nu_{x}-2 \nu_{y}=0$. While the beam loss around the injection energy was less there, a significant beam loss occurred for the latter half of the acceleration period, as shown by the black curve in Fig. 26. The Laslett tune shift for such a beam intensity with no beam painting is estimated to reach -0.2 at the end of the injection process. Most of the beam particles, therefore, successfully stay in a stable region far enough from the half integer for the early stage of the acceleration. However, the space-charge tune shift shrinks toward the bare working point as the acceleration proceeds. In addition, the tune spread due to the momentum distribution becomes significant gradually during acceleration, because the effect of the chromaticity correction with static values for the sextupoles at the injection energy shrinks during acceleration. In this operation, therefore, a part of

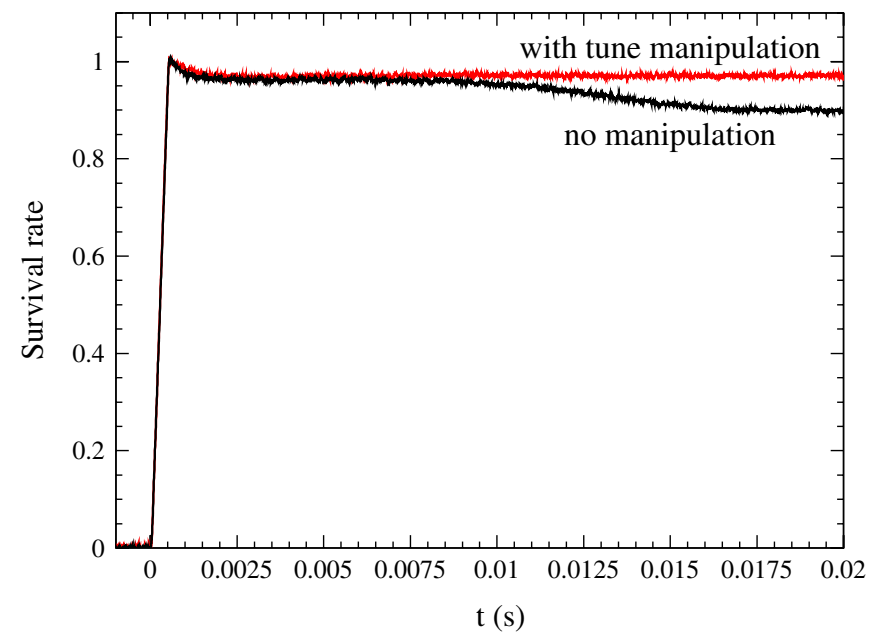

FIG. 26. (Color) Beam survival rates as a function of the revolution time measured with no beam painting scheme using DCCT, where $5 \times 10^{12}$ protons per bunch were injected to the RCS. The beam loss observed for the latter half of the acceleration period was improved by the tune variation shown in Fig. 27.

the beam is caught in the half integer line at the middle and late stage of the acceleration period, leading to the observed particle loss.

In order to overcome such a situation, the magnetic field tracking for the main quadrupole magnets was manipulated so that the bare working point moved away from the half integer during acceleration, as shown in Fig. 27, where the top of the excitation current pattern for two families of

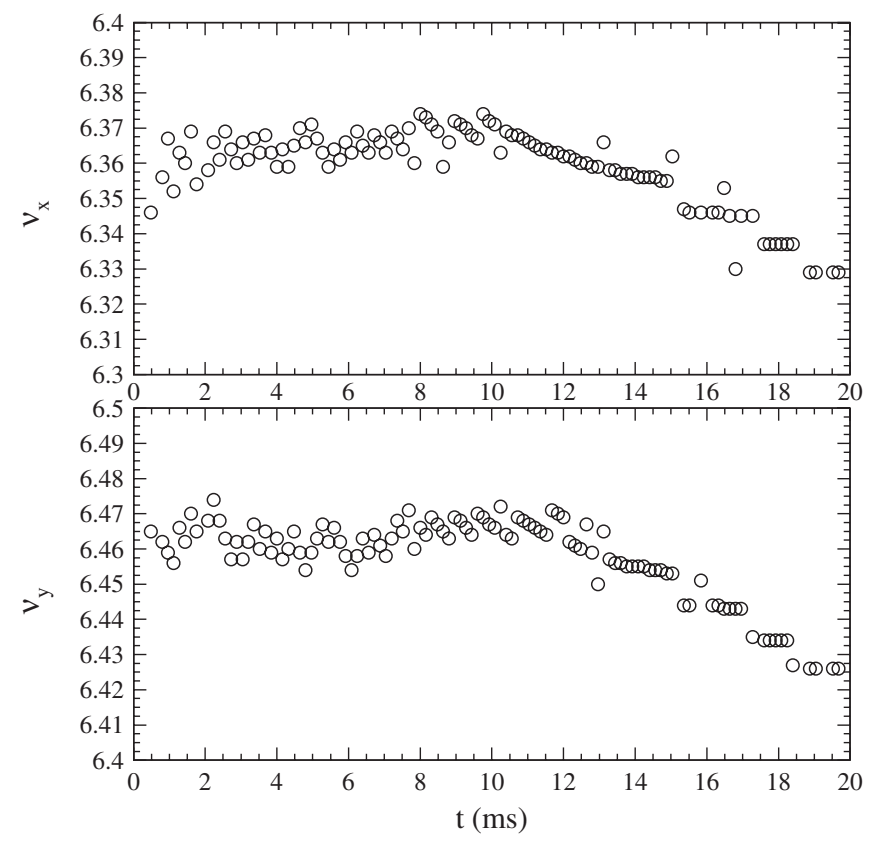

FIG. 27. Horizontal (upper) and vertical (lower) tune variations measured over the acceleration process, where the magnetic field tracking for the quadrupole magnets was manipulated. 
quadrupole magnets (QFM and QDX) placed in the dispersion-free long straight section was lowered by adjusting their $\mathrm{AC}$ and $\mathrm{DC}$ components. As shown by the red curve in Fig. 26, the beam loss observed for the latter half of the acceleration period disappeared for the improved tune variation.

For higher beam-power operations, the space-charge tune shift is still significant even with a beam painting scheme, and brings a strong limitation on the betatron tunability. This result demonstrates that we can make the most of the betatron tune space interrupted by various resonance lines and cure the beam loss by dynamically controlling the bare working tune according to the magnitude and footprint of the space-charge tune shift.

\section{G. Beam intensity loss at the early stage of acceleration}

Figure 28 shows beam intensity losses in the first $3 \mathrm{~ms}$ measured for the same intensity beam as that in Fig. 26 with no beam painting scheme in the $181 \mathrm{MeV}$ beam storage mode, where the extraction DC magnets were excited at $181 \mathrm{MeV}$ or $3 \mathrm{GeV}$. As clearly shown in the figure, it was confirmed that the beam intensity loss observed at the early stage of acceleration in Fig. 26 was mainly caused by the static leakage fields from the extraction beam line discussed in the last section.

With the current injection energy of $181 \mathrm{MeV}$, we aim at providing at least $0.3 \mathrm{MW}$ output beam power. The permissible range of intensity loss in this operation, which is determined by the current collimator capacity of $4 \mathrm{~kW}$, is $22 \%$ at the injection energy. After upgrading the injection energy to $400 \mathrm{MeV}$, we will drive for $1 \mathrm{MW}$ output beam power, in which the allowable intensity loss is $3 \%$ if

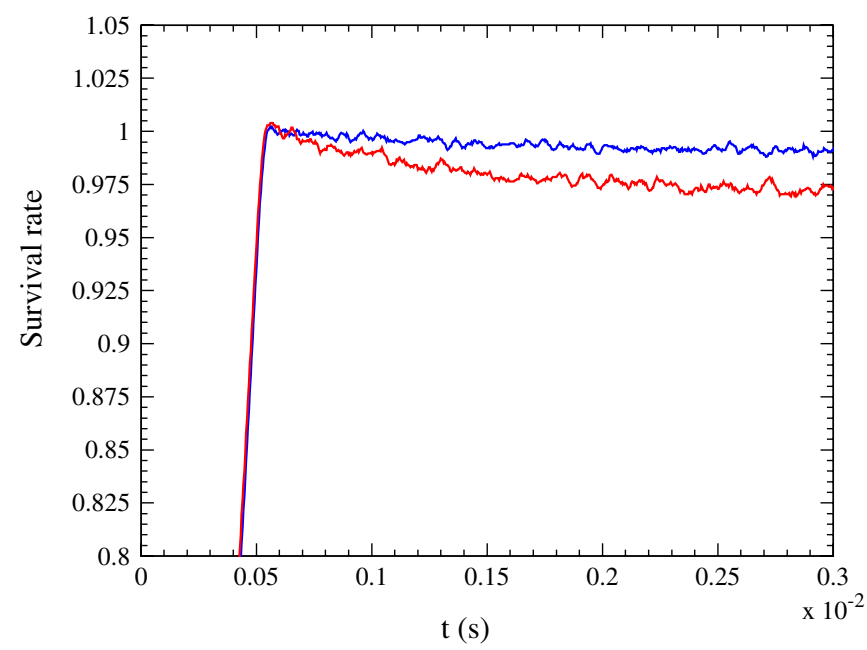

FIG. 28. (Color) Beam survival rates in the first $3 \mathrm{~ms}$ measured for the same intensity beam as that in Fig. 26 with no beam painting scheme in the $181 \mathrm{MeV}$ beam storage mode, where the extraction DC magnets were excited at $181 \mathrm{MeV}$ (blue curve) or $3 \mathrm{GeV}$ (red curve). assuming the same collimator limit at the injection energy. The above two operations give an equivalent space-charge effect at each injection energy. Therefore, to achieve 0.3 MW output with less than $3 \%$ intensity loss for $181 \mathrm{MeV}$ injection energy is the first matter leading to the realization of $1 \mathrm{MW}$ output with $400 \mathrm{MeV}$ injection energy. For this goal, first, it is essential to shield the leakage fields from the extraction beam line, and then we will try further beam loss reduction for higher intensity beams by making the most of the beam painting scheme.

\section{PRELIMINARY TEST OF THE BEAM PAINTING}

We performed a preliminary test of the transverse and longitudinal painting injection schemes in the beam storage mode using a linac beam with the design pulse length ( $5 \mathrm{~mA}$ peak, $0.5 \mathrm{~ms}$ long, and typically $26 \%$ chopper beamon duty factor, corresponding to $4.2 \times 10^{12}$ per bunch in the single-bunch operation at the RCS) in the run cycles in May-June 2008.



FIG. 29. (Color) Horizontal (left) and vertical (right) beam profile mountain views measured over the injection period $(0.5 \mathrm{~ms})$ with IPM, where the upper ones show those for the center injection, and the lower ones are with the transverse beam painting (correlated painting). The painting emittance was set at $150 \pi \mathrm{mm}$ mrad for both the horizontal and vertical planes. 
In the transverse beam painting, PBH1-4 and PBV1-2, which make a time dependent injection-orbit bump at the first stripper foil, were used as well as SB1-4 (see Fig. 2). In the horizontal plane, the injection beam was painted from the middle to the outside of the RCS beam ellipse along its major axis in phase space by sweeping the closed orbit with PBH1-4 installed on both sides of SB1-4, while in the vertical plane, the injection beam was moved from the middle to the outside (correlated painting) or from the outside to the middle (anticorrelated painting) along the $y^{\prime}$ axis using PBV1-2 installed most downstream of L-3BT nearly 180 degrees away from the first foil in betatron phase advance. Figure 29 shows beam profile mountain views measured with the IPM for the center (upper) and correlated painting (lower) injections, where the painting emittance was set at $150 \pi \mathrm{mm}$ mrad for both the horizontal and vertical planes $(216 \pi \mathrm{mm}$ mrad in the design). A series of tuning procedures for the beam painting, such as to identify a footprint of the phase space coordinates of the injected beam on the RCS beam ellipse over the painting process (applied technique of the injection error correction) and to change the painting area, was successfully demonstrated in the beam storage mode $[30,36]$.

On the other hand, the longitudinal beam painting was performed by combination of the momentum-offset injec-

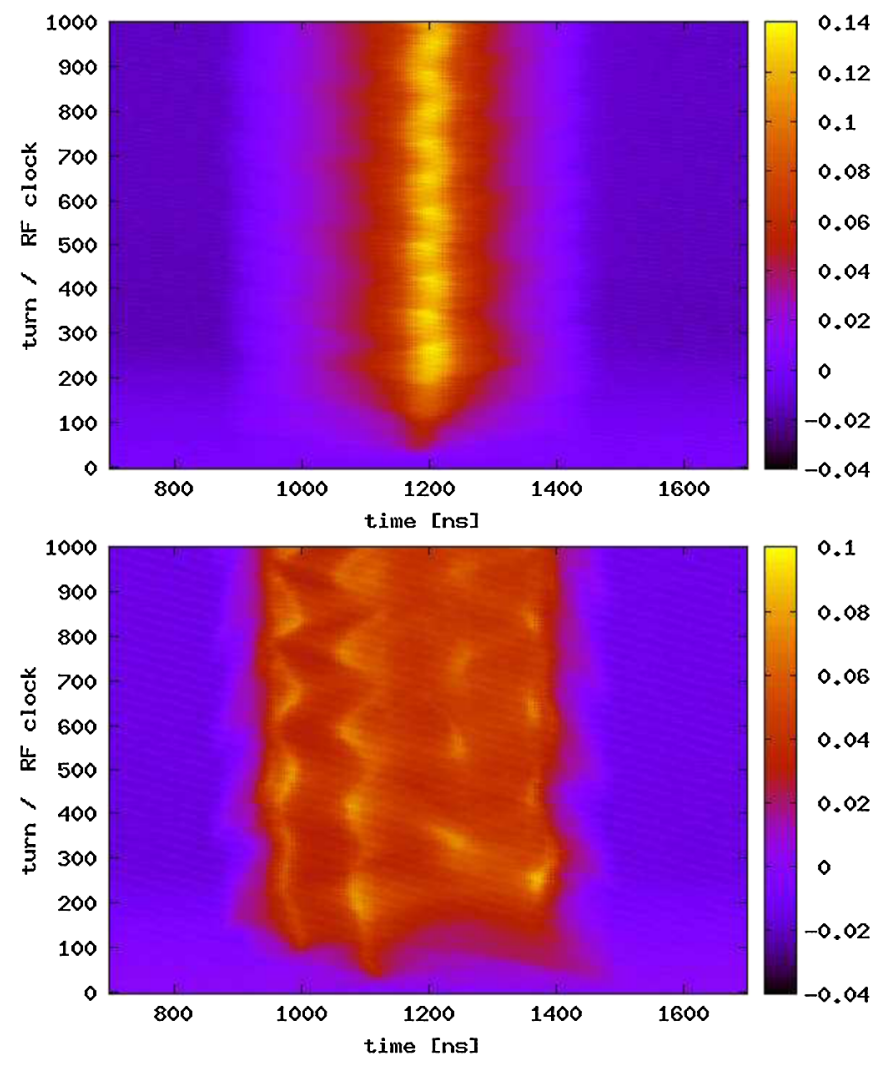

FIG. 30. (Color) Longitudinal beam profile mountain views measured for the injection period $(0.5 \mathrm{~ms})$ using WCM without (upper) and with (lower) the longitudinal beam painting. tion scheme, in which the rf frequency had an offset, and superposing a second harmonic rf voltage $(h=4)$ to widen and flatten the rf bucket [37]. The phase sweep of the second harmonic rf voltage relative to the fundamental one $(h=2)$ was also employed so that the shape of the rf bucket was dynamically changed during the injection process. For improving and preventing the degradation of the bunching factor, the longitudinal motion was surveyed for various parameters [38] preliminarily in the beam storage mode. Figure 30 shows longitudinal beam profile mountain views measured using WCM without (upper) and with (lower) the longitudinal painting. In this case of longitudinal painting, the second harmonic component has an amplitude of $80 \%$ of the fundamental voltage, and its phase is sweeping from -60 to 0 degrees. The $\mathrm{rf}$ frequency offset corresponds to a $-0.2 \%$ momentum offset. Figure 30 clearly shows that the longitudinal beam profile is well widened uniformly with the applied manipulations.

While the beam painting scheme is very effective to control the charge density of the beam and mitigate the space-charge effect, it logically increases the number of particles with large amplitudes for both the transverse and longitudinal planes, possibly causing some issues coming from their nonlinear behavior. In order to gain a sufficient ability of the beam painting scheme, it is essential to fulfill the global optimization for various correlated parameters, such as to search a bare working tune with a dynamic aperture large enough for the painting emittance especially around the injection period, to optimize a tune variation over the acceleration process according to magnitude and footprint of the space-charge tune depression, and to get a better balance of the transverse and longitudinal painting areas. For higher beam-power operations, the beam painting scheme will be a key to handle the beam loss especially at the injection through the early stage of the acceleration. The transverse painting also plays an important role in terms of mitigating a pressure wave on the neutron target $[39,40]$. In the second commissioning stage, our efforts will be dedicated to such a global parameter optimization including the beam painting scheme toward higher beampower operations.

\section{CURRENT MACHINE ACTIVATION}

A residual radiation survey for the accelerator components has been performed every beam run, where their radiation level is measured with contact on the vacuum chamber typically a half day after the beam shutdown. Here we show a result of the residual radiation survey performed after the beam run in February 2008. In this run, high beam-power demonstrations, mentioned in the last section, were carried out, and the total number of particles injected to the RCS amounted to $3 \times 10^{16}$, most of which come from the high beam-power demonstration performed at $25 \mathrm{~Hz}$ for four minutes. 
The most significant levels of residual radiation were observed around the injection section, especially downstream of the first stripper foil (see Fig. 2). The highest level detected in this area was $120 \mu \mathrm{Sv} / \mathrm{h}$ at the entrance of the collimator section, which corresponds to a somewhat narrow section. The other radiation levels in this area were $1.2 \mu \mathrm{Sv} / \mathrm{h}$ or less upstream of the first foil and $2.2-16 \mu \mathrm{Sv} / \mathrm{h}$ downstream of it. In the injection area, there are many possible sources of particle loss such as Lorentz stripping, decay of excited $\mathrm{H}^{0}$, and foil scattering, in which the most significant beam loss source is the scattering on the first stripper foil. So far, the center injection has been typically performed instead of the painting injection, in which the circulating beam hits the foil every turn during injection. In addition, the current fall time of the injection-orbit bump $(0.5 \mathrm{~ms})$ is longer than the design $(0.18 \mathrm{~ms})$, and also the size and positioning of the foil is still not optimized. These circumstances increase the frequency of the foil hitting. The typical number of foil hits in the current situation is calculated to be 150 . It is 8 times larger than the design, and its resultant beam loss rate during the injection is calculated to be $1.5 \%$ considering both Coulomb and nuclear scatterings [30,41]. Most of the lost particles should be localized on the collimator, but some of them with large scattering angles, which are mainly from the nuclear scattering and estimated to be $0.5 \%$ of the lost particles, lead to uncontrolled beam losses downstream of the first foil. Most of the residual radiations observed downstream of the first foil probably come from such a large-angle foil scattering. The foil scattering also contributes to halo formation of the circulating beam. Therefore it also ought to contribute to particle losses at the early stage of the acceleration process after the beam injection is complete. For realizing higher beam-power operations, further machine parameter optimizations to reduce the foil hitting probability are also essential.

While some measurable residual radiation was detected also at the missing-bend cells with a horizontal dispersion peak, they mainly come from beam losses during the rf tuning and the longitudinal studies. In the extraction section, no significant radiation was detected.

So far, there has been no critical machine activation and it looks like most of particle losses are well localized on the collimators [42]. However, we still do not have enough data on the residual radiation for stable beam operations, and cannot yet make a reliable extrapolation for the machine activation, because we have been in the commissioning phase so far and the beam conditions changed often according to the beam tuning and study items mainly in the beam-on-demand operation. First 12- or 24-hour stable beam operations for the MLF are planned with $4 \mathrm{~kW}$ output beam power in September 2008. Then the beam power will increase to $20 \mathrm{~kW}$ in December 2008 and to $100 \mathrm{~kW}$ early after April 2009. We must hereafter proceed with a careful beam-power ramp-up plan including monitoring and trending the residual radiation levels.

\section{SUMMARY}

The RCS was beam commissioned in October 2007. The initial beam tuning and underlying beam studies through various beam-dynamics measurements such as optical functions, turn-by-turn beam positions, and transverse and longitudinal beam profiles were completed in February 2008, in which we successfully demonstrated a consecutive $52 \mathrm{~kW}$ output $\left(4.3 \times 10^{12}\right.$ protons per pulse $)$ in the one-bunch operation at $25 \mathrm{~Hz}$, and also two-bunch extraction of $1.1 \times 10^{13}$ protons per pulse in the beamon-demand operation (corresponding to $130 \mathrm{~kW}$ output if performing $25 \mathrm{~Hz}$ repetition). The beam painting scheme, which is a key for ramping up the beam intensity, has also been well demonstrated in the recent run cycles in MayJune 2008. While several beam dynamics and technical issues were found, our understanding of lattice properties and various motions of the beam especially related to the underlying single-particle nature at low intensities was deepened through the beam commissioning process. The emphasis of the RCS beam tuning hereafter will be focused on higher beam-power operations with better stability.

\section{ACKNOWLEDGMENTS}

The authors would like to acknowledge the efforts of the entire J-PARC project staff for their continuous work in designing, fabricating, installing, and checking out this machine. We also acknowledge linac, MR, and MLF group members for their indispensable support during the commissioning. The authors are grateful to Professor K. Oide and KEKB group members for lots of helpful advice on the beam commissioning and to Dr. S. Machida for his valuable work in the design stage of the RCS. Finally, we would like to express our special thanks to the late Professor J. Kishiro, who was the RCS group leader.

[1] Accelerator Technical Design Report for High-Intensity Proton Accelerator Facility Project, JAERI Report No. JAERI-Tech 2003-044 and KEK Report No. 2002-13.

[2] M. Ikegami, in Proceedings of the Advanced Beam Dynamics Workshop on High-Intensity, High-Brightness Hadron Beams (HB2008), Nashville, Tennessee, 2008, WGD02.

[3] M. Ikegami, in Proceedings of the Advanced Beam Dynamics Workshop on High-Intensity, High-Brightness Hadron Beams (HB2008), Nashville, Tennessee, 2008 (Ref. [2]), WGB08.

[4] T. Koseki, in Proceedings of the Advanced Beam Dynamics Workshop on High-Intensity, High-Brightness Hadron Beams (HB2008), Nashville, Tennessee, 2008 (Ref. [2]), WGD11.

[5] H. Ao, K. Hirano, A. Ueno, T. Morishita, K. Hasegawa, Y. Yamazaki, M. Ikegami, and V. Paramonov, in Proceedings of the 2007 Particle Accelerator 
Conference, Albuquerque, New Mexico, 2007 (IEEE, Albuquerque, New Mexico, 2007), pp. 1514-1516.

[6] N. Tani, T. Adachi, S. Igarashi, Y. Watanabe, H. Someya, H. Sato, and J. Kishiro, IEEE Trans. Appl. Supercond. 14, 409 (2004).

[7] Y. Watanabe, T. Adachi, H. Someya, S. Koseki, and S. Ogawa, IEEJ Trans. IA 126, 681 (2006).

[8] M. Yoshimoto, P. K. Saha, F. Noda, J. Kamiya, T. Takayanagi, M. Watanabe, O. Takeda, M. Kinsho, and Y. Irie, in Proceedings of the 10th European Particle Accelerator Conference, Edinburgh, Scotland, 2006 (EPS-AG, Edinburgh, Scotland, 2006), pp. 1765-1767.

[9] K. Yamamoto and M. Kinsho, in Proceedings of the 21st Particle Accelerator Conference, Knoxville, 2005 (IEEE, Piscataway, NJ, 2005), pp. 1365-1367.

[10] M. Yoshii, S. Anami, E. Ezura, K. Hara, Y. Hashimoto, C. Ohmori, A. Takagi, M. Toda, K. Haga, K. Hasegawa, M. Nomura, A. Schnase, F. Tamura, and M. Yamamoto, in Proceedings of the 2007 Particle Accelerator Conference, Albuquerque, New Mexico, 2007 (Ref. [5]), pp. 15111513.

[11] K. Yamamoto, M. Kinsho, F. Noda, Y. Irie, and M. Shirakata, in Proceedings of the 19th Particle Accelerator Conference, Chicago, Illinois, 2001 (IEEE, Piscataway, NJ, 2001), pp. 3615-3617.

[12] H. Hotchi, F. Noda, N. Tani, J. Kishiro, S. Machida, and A. Yu. Molodojentsev, in Proceedings of the 21st Particle Accelerator Conference, Knoxville, 2005 (Ref. [9]), pp. 916-918.

[13] H. Hotchi, F. Noda, Y. Irie, S. Machida, and A. Yu. Molodojentsev, in Proceedings of the 10th European Particle Accelerator Conference, Edinburgh, Scotland, 2006 (Ref. [8]), pp. 2104-2106.

[14] N. Hayashi, S. Hiroki, R. Saeki, R. Toyokawa, K. Yamamoto, M. Yoshimoto, D. Arakawa, S. Hiramatsu, S. Lee, K. Satou, M. Tejima, and T. Toyama, in Proceedings of the 11th European Particle Accelerator Conference, Genoa, 2008 (EPS-AG, Genoa, Italy, 2008), pp. 1125-1127.

[15] S. Hiroki, N. Hayashi, M. Kawase, F. Noda, P. K. Saha, H. Sako, H. Takahashi, A. Ueno, Y. Arakida, S. Lee, and T. Toyama, in Proceedings of the 11th European Particle Accelerator Conference, Genoa, 2008 (Ref. [14]), pp. 1131-1133.

[16] H. Sako, S. Hiroki, H. Takahashi, K. Yamamoto, and H. Ikeda, in Proceedings of the 11th European Particle Accelerator Conference, Genoa, 2008 (Ref. [14]), pp. 1272-1274.

[17] N. Hayashi, S. Hiroki, R. Saeki, R. Toyokawa, K. Yamamoto, D. Arakawa, S. Hiramatsu, S. Lee, K. Satou, M. Tejima, and T. Toyama, in Proceedings of the 11th European Particle Accelerator Conference, Genoa, 2008 (Ref. [14]), pp. 1128-1130.

[18] H. Takahashi, N. Hayashi, and M. Sugimoto, in Proceedings of the 11th European Particle Accelerator Conference, Genoa, 2008 (Ref. [14]), pp. 1553-1555.

[19] K. Satou, S. Lee, T. Toyama, M. Tejima, N. Hayashi, A. Ueno, K. Yamamoto, R. Saeki, and H. Harada, in Proceedings of the 11th European Particle Accelerator Conference, Genoa, 2008 (Ref. [14]), pp. 1275-1277.

[20] S. Lee, M. Tanaka, T. Toyama, and J. Kishiro, in
Proceedings of the 9th European Particle Accelerator Conference, Lucerne, 2004 (EPS-AG, Lucerne, 2004), pp. 2667-2669.

[21] H. Yoshikawa, H. Sakaki, H. Sako, H. Takahashi, G-B. Shen, Y. Kato, Y. Itoh, H. Ikeda, T. Ishiyama, H. Tsuchiya, S. Sawa, M. Kawase, M. Sugimoto, T. Suzuki, K. Watanabe, S. Fukuda, M. Adachi, S. Hatakeyama, A. Akiyama, T. Katoh, N. Kamikubota, K. Kudo, T. Matumoto, H. Nakagawa, J. Odagiri, Y. Takeuchi, M. Tanaka, N. Yamamoto, T. Iitsuka, S. Motohashi, M. Takagi, and S. Yoshida, in Proceedings of the 2007 International Conference on Accelerator and Large Experimental Physics Control Systems, Knoxville, Tennessee, 2007, pp. 62-64.

[22] H. Sako, A. Ueno, T. Ohkawa, Y. Kondo, T. Morishita, M. Ikegami, and H. Akikawa, in Proceedings of the 2008 Linac Conference, Victoria, Canada, 2008, MOP078.

[23] T. Takayanagi, J. Kamiya, M. Watanabe, Y. Yamazaki, Y. Irie, J. Kishiro, I. Sakai, and T. Kawakubo, IEEE Trans. Appl. Supercond. 16, 1358 (2006).

[24] T. Takayanagi, K. Kanazawa, T. Ueno, H. Someya, H. Harada, Y. Irie, M. Kinsho, Y. Yamazaki, M. Yoshimoto, J. Kamiya, M. Watanabe, M. Kuramochi, and K. Satou, IEEE Trans. Appl. Supercond. 18, 306 (2008).

[25] I. Sugai, Y. Takeda, M. Oyaizu, H. Kawakami, Y. Irie, K. Hara, A. Takagi, H. Hattori, K. Kawasaki, J. Kamiya, and M. Kinsho, in Proceedings of the 10th European Particle Accelerator Conference, Edinburgh, Scotland, 2006 (Ref. [8]), pp. 1753-1755.

[26] M. Yoshimoto, T. Ueno, T. Togashi, O. Takeda, K. Kanazawa, M. Watanabe, Y. Yamazaki, J. Kamiya, T. Takayanagi, M. Kuramochi, M. Kinsho, Y. Irie, H. Hujimori, S. Igarashi, and H. Nakayama, IEEE Trans. Appl. Supercond. 18, 297 (2008).

[27] M. Watanabe, J. Kamiya, M. Kinsho, K. Hirano, T. Takayanagi, Y. Yamazaki, M. Yoshimoto, and Y. Irie, in Proceedings of the 11th European Particle Accelerator Conference, Genoa, 2008 (Ref. [14]), pp. 3676-3678.

[28] S. Igarashi, J-PARC Accelerator Memo No. 7 (2008).

[29] See http://acc-physics.kek.jp/SAD/sad.html.

[30] P. K. Saha, in Proceedings of the Advanced Beam Dynamics Workshop on High-Intensity, High-Brightness Hadron Beams (HB2008), Nashville, Tennessee, 2008 (Ref. [2]), WGC05.

[31] M. Yoshimoto, J. Kamiya, M. Watanabe, T. Takayanagi, T. Togashi, T. Ueno, M. Kuramochi, H. Hotchi, P. K. Saha, H. Harada, and M. Kinsho, in Proceedings of the 11th European Particle Accelerator Conference, Genoa, 2008 (Ref. [14]), pp. 3626-3628.

[32] F. Tamura, A. Schnase, M. Nomura, M. Yamamoto, K. Hasegawa, K. Haga, M. Yoshii, C. Ohmori, M. Toda, K. Hara, E. Ezura, S. Anami, and A. Takagi, in Proceedings of the 11th European Particle Accelerator Conference, Genoa, 2008 (Ref. [14]), pp. 364-366.

[33] H. Hotchi, F. Noda, and S. Machida, in Proceedings of the 2007 Particle Accelerator Conference, Albuquerque, New Mexico, 2007 (Ref. [5]), pp. 4078-4080.

[34] J. Kamiya, T. Takayanagi, T. Kawakubo, S. Murasugi, and E. Nakamura, in Proceedings of the 21st Particle Accelerator Conference, Knoxville, 2005 (Ref. [9]), pp. 1009-1011. 
[35] J. Kamiya, M. Kinsho, M. Kuramochi, T. Takayanagi, T. Togashi, T. Ueno, M. Watanabe, and M. Yoshimoto, in Proceedings of the 11th European Particle Accelerator Conference, Genoa, 2008 (Ref. [14]), pp. 3590-3592 [Phys. Rev. ST Accel. Beams (to be published)].

[36] H. Harada, Ph.D. thesis, Hiroshima University, 2009.

[37] F. Tamura, A. Schnase, and M. Yoshii, Phys. Rev. ST Accel. Beams 11, 072001 (2008).

[38] M. Yamamoto, S. Anami, E. Ezura, K. Hasegawa, K. Hara, K. Horino, M. Nomura, C. Ohmori, A. Schnase, T. Shimada, H. Suzuki, K. Takata, A. Takagi, F. Tamura, M. Toda, and M. Yoshii, in Proceedings of 5th Annual Meetings of Particle Accelerator Society of Japan, Hiroshima, Japan, 2008, WP039.
[39] S. Meigo, F. Noda, S. Ishikura, M. Futakawa, S. Sakamoto, and Y. Ikeda, Nucl. Instrum. Methods Phys. Res., Sect. A 562, 569 (2006).

[40] M. Futakawa, T. Wakui, H. Kogawa, and Y. Ikeda, Nucl. Instrum. Methods Phys. Res., Sect. A 562, 676 (2006).

[41] P. K. Saha, F. Noda, H. Hotchi, Y. Irie, and S. Machida, in Proceedings of the 2007 Particle Accelerator Conference, Albuquerque, New Mexico, 2007 (Ref. [5]), pp. 15261528.

[42] K. Yamamoto, in Proceedings of the Advanced Beam Dynamics Workshop on High-Intensity, High-Brightness Hadron Beams (HB2008), Nashville, Tennessee, 2008 (Ref. [2]), WGC10. 